\title{
Spatial averaging of streamwise and spanwise velocity measurements in wall-bounded turbulence using $\vee$ - and $\times$-probes
}

\author{
Jimmy Philip, Rio Baidya, Nicholas Hutchins, Jason P Monty \\ and Ivan Marusic \\ Department of Mechanical Engineering, The University of Melbourne, Parkville, VIC \\ - 3010, Australia.
}

E-mail: jimmyp@unimelb.edu.au or jimmy.philip@gmail.com

\begin{abstract}
The effect of finite-dimensions of $\vee$ - and $\times$-probes are investigated for the measurement of mean and variances of streamwise and spanwise velocities in wallturbulence. The probes are numerically simulated using a Direct Numerical Simulation database of channel flow at friction Reynolds number $\left(R e_{\tau}\right)$ of 934 by varying the probe parameters, namely, the wire-lengths $(l)$, the angle between the wires $(\theta)$ and the spacing between the wires $(\Delta s)$. A single inclined wire is first studied to isolate the effect of $l$ and $\theta$. Analytical expressions for the variances of the streamwise and spanwise velocities are derived by applying a linear-box type filter to the unfiltered velocity field for both $\vee$ - and $\times$-probes (at $\theta=45^{\circ}$, and arbitrary $l$ and $\Delta s$ ). A similar expression for the streamwise variance in the case of a single inclined wire (for arbitrary $l$ and $\theta$ ), is also derived. These analytical expressions, supplemented with a model for the correlation over the wire-length compare favorably with the numerical simulation results, and more importantly explain various trends that are observed in the variances with varying parameters. Close to the wall (where the errors are generally higher) the errors in spanwise variances of the $\times$-probes are much lower than the $\vee$-probes, owing to an 'error-cancelling' mechanism present in $\times$-probes due to the effect of $l$ and $\Delta s$, as well as due to the procedure of recovering the velocities from two wires. The errors in the streamwise variances are comparable for both $\vee$ - and $\times$-probes. On the other hand, mean velocities are measured with almost no error by the $\vee$-probe, whereas the $\times$-probe induces finite errors in mean velocities due to the fact that the two wires experience different mean velocities in $\times$-probes unlike $\vee$-probes. These results are explained using the corresponding analytical results, which also show that under the effect of a linear filter, measured variances depend only on the fluctuating velocities (more precisely on the two-point correlations) and the measured means depend only on the unfiltered mean. The various results are found to be in accordance with the experimental measurements carried out in a turbulent boundary layer at $R e_{\tau} \approx 5000$. Finally, considering the physical positioning of wires in the $\vee$ - and $\times$-probes combined with the above results suggests that $\times$-probes might be more suitable for streamspanwise velocity measurements than the $\vee$-probes in conventional wind-tunnels.
\end{abstract}




\section{Introduction}

The finite-dimension effect of the two-wire probes for the measurement of streamwise and spanwise velocities $(u, v$, respectively) in wall-turbulence is investigated. The present work extends the study of single hot-wires presented in Philip et al. [2013] to $\vee$-probes and $\times$-probes for streamwise and spanwise velocities. However, unlike single-wires where the effect of finite-dimension was only spatial 'averaging', in the two-wire scenario apart from the spatial averaging there is also the adverse effect of the separation between the two wires, not to mention the different inclination (or cone) angles at which the wires are placed with respect to the mean flow.

Cross-wires sensors have remained important for multi-component velocity measurements in turbulent flows even with the recent advances in Particle Image Velocimetry. One of the challenges with cross-wires is ensuring that the relative velocity vectors of the flow being measured do not exceed a critical cone or wire angle of the sensor [Marusic and Perry, 1995]. Exceedingly large relative velocity vector angles lead to errors and this can only be overcome by reducing the relative vector angle, using for example flying hot-wires, or by increasing the angle between the two wires. Zero-pressure-gradient (ZPG) flows pose less challenges to the cross-wires in terms of the cones angles, which become more critical in adverse-pressure-gradient (APG) flows [e.g., Marusic and Perry, 1995]. The standard wire angles of $\pm 45^{\circ}$ are sufficient for ZPG and mild APG flows, whereas large wire angles up to $\pm 60^{\circ}$ have been employed in severe APG as well as rough wall flows along with flying hot-wires [e.g., Perry et al., 1987, Krogstad and Skåte, 1995, Marusic and Perry, 1995]. Flying hot-wires have also been used in turbulent jet flows to alleviate the cone angle problems [e.g., Hussein et al., 1996, Nickels and Perry, 1996]. The effects of wire-separation combined with wire-length effect on the turbulence statistics has not been investigated equally well. Notable exceptions are the studies employing Direct Numerical Simulation (DNS) databases, wherein the effect of various parameters can be studied independently. Moin and Spalart [1987] marks the first study of its kind at $R e_{\tau}$ of 180 (where, $R e_{\tau}=u_{\tau} \delta / \nu$, and $\delta$ is the turbulent boundary layer thickness or channel half-height, $u_{\tau}$ the friction velocity and $\nu$ the kinematic viscosity). A more detailed investigation was undertaken by Suzuki and Kasagi [1992] at $R e_{\tau}=150$ for both single and cross-wire, however concentrating primarily on the effect of separation rather than spatial averaging for cross-wires. More recently Vukoslavčević and Wallace [2011] have tried to understand the errors in a three or four wire sensor at $R e_{\tau}=200$. Hot-wire spatial resolution issues for single normal wires have been investigated extensively and the recommendations of Ligrani and Bradshaw [1987] are considered to be reasonable for turbulent boundary layer measurements. No such extensive study exists for cross-wires. A partial aim of the present investigation is to fill this void using the DNS database of a turbulent channel flow simulation at $R e_{\tau}=934$.

The study of two-wire-probes can be separated into two different categories depending on the variation of mean velocity encountered by the sensors: those on the 


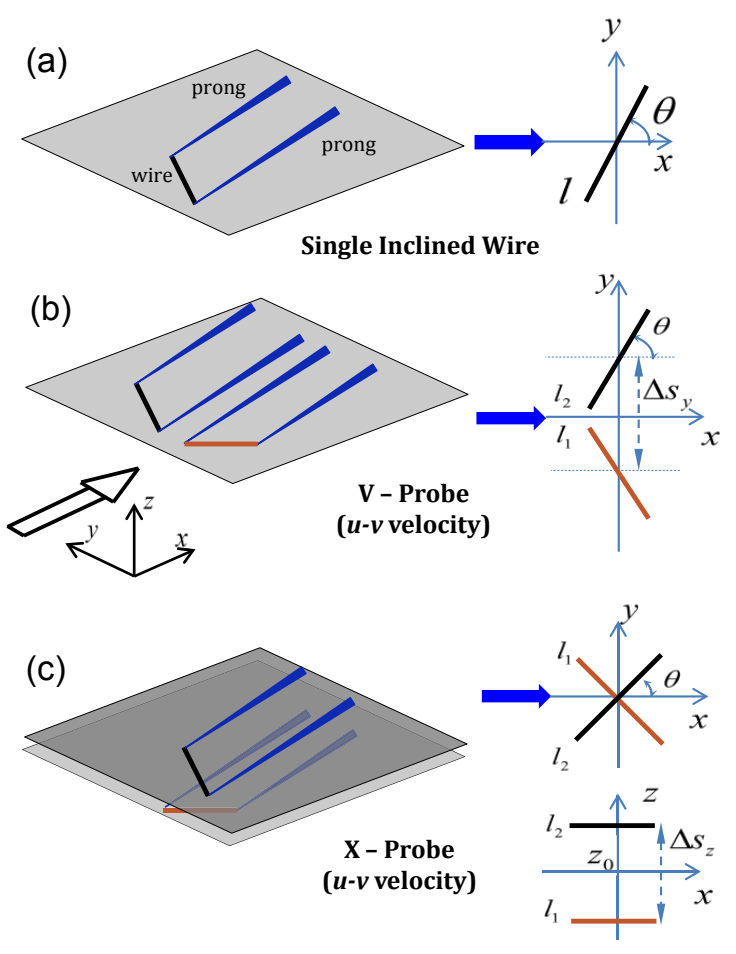

Figure 1. Sensors in the homogenous plane/s (i.e., fixed $z$-location/s). (a) A single hot-wire with the wire placed in the spanwise direction. (b) A $\vee$-probe for the measurement of $u-v$ (streamwise-spanwise) velocity. (c) An $\times$-probe, again for $u-v$ velocity.

homogeneous plane/s (or the horizontal $x-y$ plane) and those on the inhomogeneous plane/s (or the vertical $x z$-plane). The steamwise, spanwise and wallnormal directions are denoted by $x, y$ and $z$, respectively, and $u, v$ and $w$ the corresponding velocities.

Here we shall investigate probes where the wires are on a single homogeneous plane corresponding to no mean flow variation on this plane; consequently there is no effect of wallnormal shear. A single hot-wire and $\vee$-probe belong to this category (shown in figure 1(b)), for $u-v$ (streamwise-spanwise) velocity measurements. The next level of complexity can be added if the sensor is located on two (or multiple) homogeneous $(x-y)$ planes. In this case even though the mean flow between the two planes is different, there is no mean flow variation over the sensor itself. The $\times$-probe commonly employed for the $u-v$ velocity measurements belongs here, and is shown schematically in figure 1(c). Figure, 1(a) is that of a single inclined wire. It is not the most common configuration, however, as we shall discuss below, it is a good starting point to understand the averaging effect of a hot-wire which is not perpendicular to the streamwise direction (i.e., unlike the usual hot-wire configuration), as well as without the additional complexity of two wires as in cross-wires. Apart from this, the inclined wires have been successfully used in the past [e.g. Bruun, 1995] and are still used occasionally for measurements in wall bounded turbulence [e.g. Vallikivi et al., 2012].

The paper is arranged in the following manner: the next section details the 
methodology employing the Direct Numerical Simulation (DNS) database. The bulk of the results are presented in section 3. Sequentially, we shall investigate single inclined wires, $\vee$-probes and $\times$-probes at a single wall normal location $\left(z^{+} \approx 15\right)$. Throughout, + denotes normalization by $\nu / u_{\tau}$ for length and $u_{\tau}$ for velocity. The effect of variation in wallnormal location $(z)$ on the mean velocity and variance is investigated in section 4. Some experimental data from custom-made $\vee$ - and $\times$-probes taken in a turbulent boundary layer at $R e_{\tau} \approx 5000$ are presented in section 5 , confirming the practicality of the numerical simulations and theoretical work. Finally, in section 6 we summarise and draw further conclusions.

\section{Methodology}

The effects of the finite dimension of the wires are investigated by simulating the probes numerically with the DNS database as well as using analytical tools.

The DNS database of del Álamo et al. [2003] at $R e_{\tau}=934$ is employed for the present study, which has a spatial discretisation of Fourier $\times$ Fourier $\times$ Chebychev with grid points $N_{x} \times N_{y} \times N_{z}=3072 \times 2304 \times 385$ in streamwise, spanwise and wallnormal directions. The computational domain is $8 \pi h$ units in the streamwise direction and $3 \pi h$ units in the spanwise direction, where $h$ is the half channel height. After de-aliasing in the Fourier domain, the equivalent resolution in real domain for streamwise and spanwise directions is $\Delta x^{+} \times \Delta y^{+} \approx 11.46 \times 5.73$. However, due to the $3 / 2$ de-aliasing rule, in the real domain the available database has a velocity field on an interpolated grid with a resolution of $\Delta x^{+} \times \Delta y^{+} \approx 7.6 \times 3.8$, and this is the resolution that is presented in the figures below, to be consistent with previous studies, e.g., Chin et al. [2009]. In the wall-normal direction, the grid spacing increases from $\Delta z^{+} \approx 0.03$ at the wall to a maximum 7.6 at the centre of the channel.

The DNS database is used to simulate single inclined wires, $\vee$ - and $\times$-probes. The details of the simulations and the assumptions involved will be presented in the next section before the results of each probe type is discussed.

\section{Wires in the homogeneous plane/s}

Sensors in the homogeneous plane are sketched in figure 1 for $u$-v-velocity measurements. Two wire configurations, $\vee$ and $\times$, are studied. The three parameters that govern the configuration of a $\vee$-probe (also indicated in figure $1(\mathrm{~b})$ ) are: (i) the wire length, $l$. (ii) the angle of inclination with respect to the $x$-axis, $\theta$; and (iii) the centre-to-centre spacing between the two wires, $\Delta s_{y}$. According to usual practice, both wires are assumed to be of equal length $\left(l_{1}=l_{2}=l\right)$ and inclined to the $x$-axis at the same angle $\theta$ about the $x$-axis, as shown in the figure. Of the three parameters, the effect of $l$ is probably best understood [e.g., Hutchins et al., 2009, Philip et al., 2013]. A single inclined wire is an appropriate candidate to study the effect of the inclination angle (without the additional effect of $\Delta s_{y}$ ). All the investigation presented in this section corresponds 


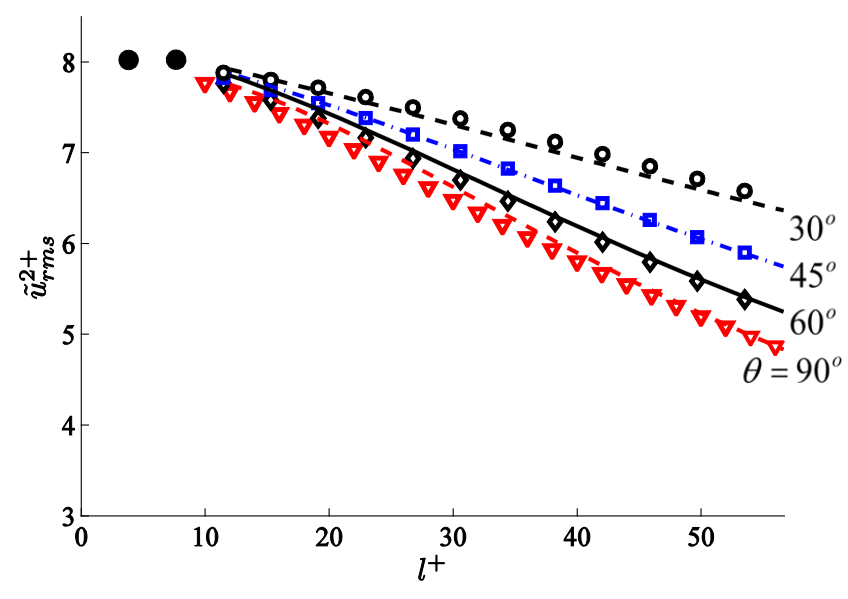

Figure 2. Attenuation of $\tilde{u}_{r m s}^{2+}$ for an inclined-wire (c.f., figure 1), at $z^{+}=15$ (a) for different wire-lengths, $l^{+}$, and inclination angles, $\theta$. Symbols are from the DNS simulations and lines are from equation (7). The two filled (black) circles correspond to the $x$ and $y$ resolution of the DNS.

to the plane nominally at $z^{+}=15$. For the single inclined wire and the $\vee$-probe, the wires lie on a single $x$-y plane, whereas for the $\times$-probe the mid-plane between the two horizontal planes where the wires lie is considered at $z^{+}=15$. Since the wires on different planes behave independently, the analysis at a single $z$-location is valuable before considering the case of varying $z$ in section 4 .

\subsection{A single inclined wire}

A single wire shown in figure $1(\mathrm{a})$ is investigated at $z^{+}=15$ for the averaging effect along its wire-length using a box-filter. The streamwise velocity, $u$, is averaged along the wire for different inclination angles $\theta$. The distribution of $u$-variance $\left(u_{r m s}^{2+}\right)$ with increasing wire length $l^{+}$, calculated using the DNS database is shown in figure 2 using symbols. Recall that $\theta=90^{\circ}$ is the same as a regular single hot-wire placed perpendicular to the incoming mean velocity. Two points are noted: for increasing $\theta$ the $u_{r m s}^{2+}$ is reduced, and the attenuation close to $\theta=90^{\circ}$ changes slowly with $\theta$, whereas there is a relatively rapid change in the attenuation values with $\theta$ for lower $\theta$.

To understand the effects due to the variations in $\theta$ we shall repeat a few results derived in Philip et al. [2013], and extend them for the current situation. For a single wire of length $l$, when the velocity signal is filtered with a generic filter, $b$ (such that the area under $b$ is equal to $l$ ), the filtered correlation is related to the unfiltered one as,

$$
\tilde{R}_{i j}(r)=\frac{1}{l^{2}} R_{i j} *\left(b_{I} \star b_{J}\right),
$$

where, $b_{I}$ and $b_{J}$ refer to the filters for $u_{i}$ and $u_{j}$, respectively, $*$ is convolution and $\star$ is correlation. The variables with indicate quantities which are filtered along with wire-length. For a single wire, measuring only $u$-velocity, $i=j=1$. If $b_{I}$ and $b_{J}$ are the same and both are assumed to be a 'box-filter' (which ranges from $-l / 2$ to $l / 2$, with the 
maximum value 1 ), i.e, the box-filter is defined as, $b(x)=1$ if $|x|<l / 2$, and $b(x)=0$ otherwise. Physically this implies that the hot-wire is equally sensitive over its entire wire-length (which is a good approximation to practical wires, where the length/dimeter ratio is kept large enough to minimise the end-conduction effects). With this the above expression simplifies to,

$$
\tilde{R}_{11}(r)=\frac{1}{l^{2}} R_{11} * \wedge_{l},
$$

where, $\wedge_{l}$ is the triangle function, which is the correlation between two box functions (and ranges from $-l$ to $l$, with the maximum value $l$ at the origin). Note that $r$ is interpreted here to be in the direction along which the hot-wire is placed, and the unfiltered correlation $R_{11}$ needs to be known along that specific direction. The most interesting quantity while studying the effects of filtering is the variance of the fluctuating velocity, $u_{r m s}^{2}$. For the filtered case, $\tilde{u}_{r m s}^{2}$ is obtained from (2) by substituting $r=0$ as,

$$
\tilde{u}_{r m s}^{2}=\tilde{R}_{11}(r=0)=\frac{2}{l^{2}} \int_{0}^{l} R_{11}(s) \cdot(l-s) \mathrm{d} s,
$$

where, $s$ is the distance along the direction of the wire.

Equation (3) can help to interpret the DNS results for attenuation in $u_{r m s}^{2}$ for different $\theta$. The expression in (3) shows that $\tilde{u}_{r m s}^{2}$ depends on the correlation along the specific $\theta$-direction. Figure $3(\mathrm{a})$ shows contours of $R_{11}\left(r_{1}, r_{2}\right)$ (the non-normalised correlation such that $R_{11}(0,0)$ is equal to the variance) on the $z^{+}=15$ plane. Also shown are the local coordinates $\left(r_{1}, r_{2}\right)$, as well as a radial coordinate system $(r, \theta)$ with $\theta=0^{\circ}$ and $90^{\circ}$ corresponding to $r_{1}$ and $r_{2}$, respectively. Since the flow is statistically stationary and homogeneous in the $x-y$ plane, $R_{11}\left(r_{1}, r_{2}\right)$ is symmetric across $r_{1}$ and $r_{2}$ making only one quarter of the distribution essential. This can be represented along the $r$ for varying $\theta$ as distributions shown in figure $3(\mathrm{~b})$.

For a fixed distance $r$, the correlation increases for decreasing $\theta$, in the range $0^{\circ} \leq \theta \leq 90^{\circ}$. This combined with equation (3) explains why the $u_{r m s}^{2}$ in figure 2 attenuates less for smaller angles. Furthermore, the correlation changes only slightly for $\theta$ increasing from $90^{\circ}$ to about $45^{\circ}$, and then there is a rapid increase. The effect of this is also evident in figure 2. Note that in the idealised single wire we have ignored the practical aspect of sensitivity of the wires at different angles to velocities, which plays a role in measurements with cross-wires.

To quantify the effect of the correlation function on the averaging for a specific wire length $l$, it is advantageous to introduce a length scale for the correlation too, which can then be compared to $l$. To this end, we model the normalised correlation, $R_{N}(r)$ $\left(=R_{11} / u_{r m s}^{2}\right)$ as,

$$
R_{N}(r, \theta)=\mathrm{e}^{-\left(r / \lambda_{\theta}\right)^{p}}
$$

where, $\lambda_{\theta}$ is a function of $\theta$ and is also the associated length scale for $R_{N}$ in a specific $\theta$-direction. The exponent $p$ needs to be chosen such that it represents the actual correlation well. Note that (c.f. figure 3(b) and the inset of figure 4) the normalised 

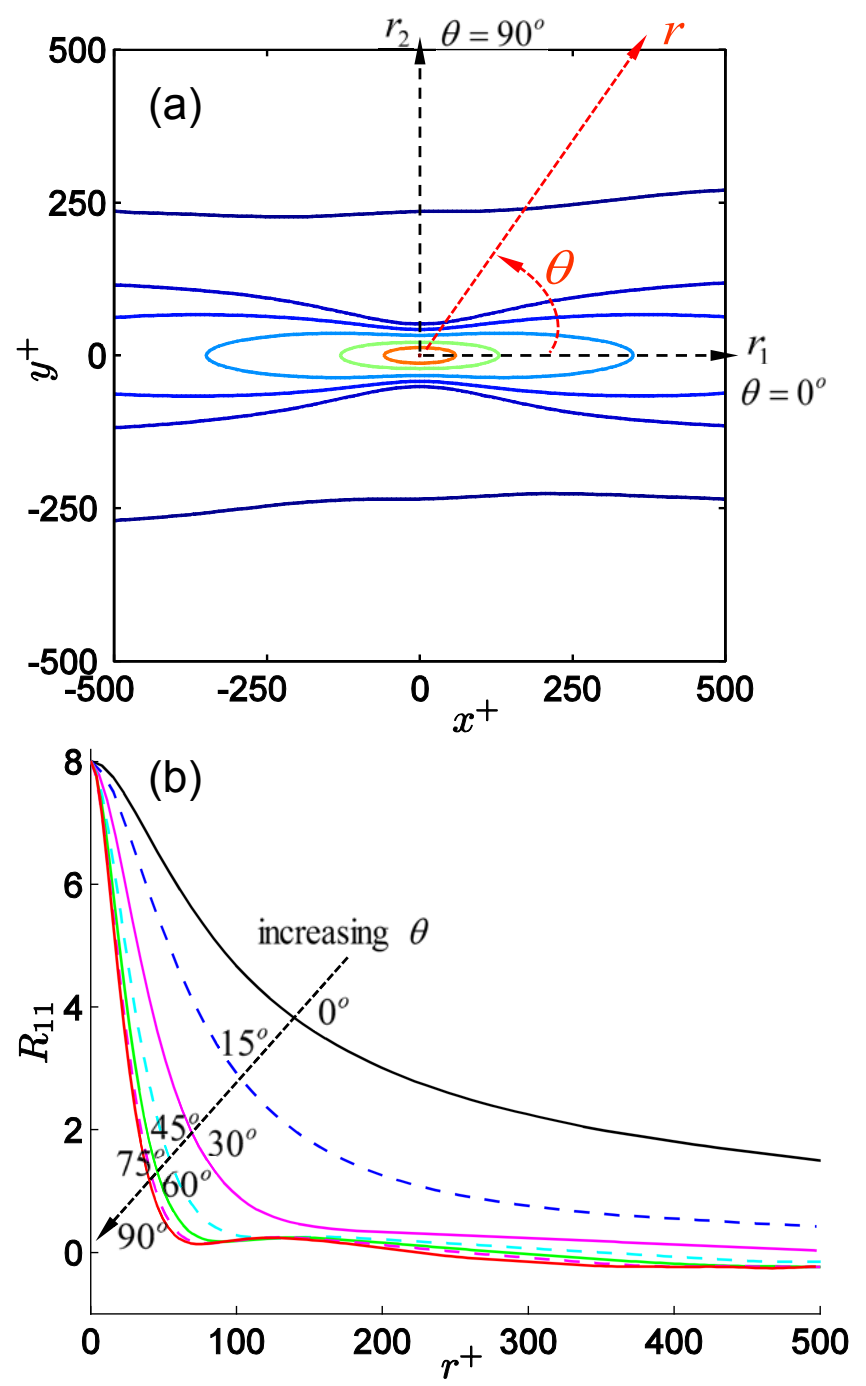

Figure 3. Two point correlation of the streamwise velocity, $R_{11}$, at $z^{+}=15$. (a) Contours of $R_{11}$ starting from the values of 8 (close to the centre) and decreasing as $6,4,2,1,0.5$ and 0 . Also shown is the definition of the radial coordinate system. (b) Explicit distributions of $R_{11}$ versus $r^{+}$with varying $\theta$.

correlation function has the shape of a Gaussian close to $\theta=90^{\circ}$ (due to the presence of the dominant near-wall streak with a spacing of 100 viscous units), whereas, close to $0^{\circ}$ it is similar to a decaying exponential. Consequently, the exponent $p$ is assumed to be,

$$
p(\theta)=1+\frac{\theta}{\pi / 2}
$$

where, $1 \leq p \leq 2$ and ensures that $R_{N}\left(r, \theta=0^{\circ}\right)=\mathrm{e}^{-\left(r / \lambda_{0} \circ\right)^{1}}$ and $R_{N}\left(r, \theta=90^{\circ}\right)=$ $\mathrm{e}^{-\left(r / \lambda_{90^{\circ}}\right)^{2}}$.

The length representing the correlation $\lambda_{\theta}$ is obtained by fitting the function (4) up to $r^{+}=100$, to the actual values presented in the inset of figure 4 . The distribution of $\lambda_{\theta}$ is presented in figure 4 for varying $\theta$. The value of $r^{+}=100$ is arbitrarily chosen 


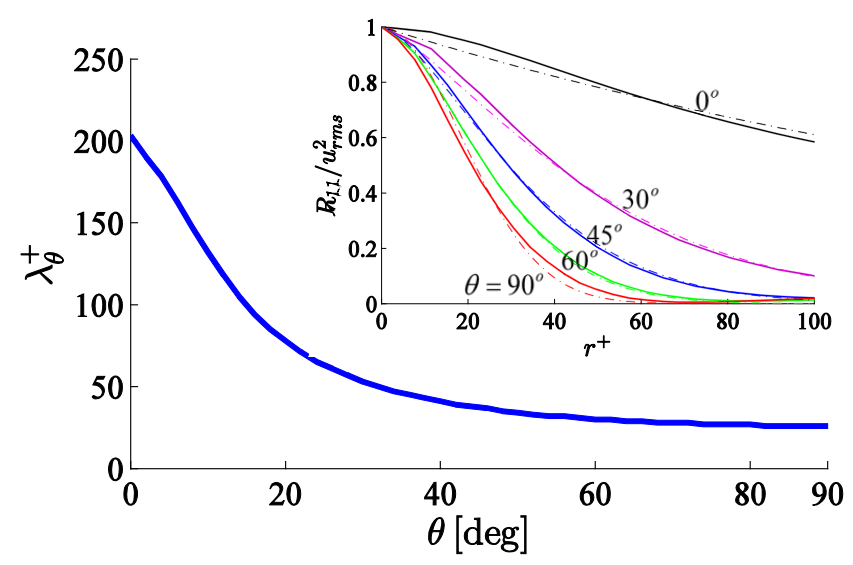

Figure 4. Distributions of $\lambda_{\theta}$ (of equation 4) with $\theta$. Inset shows the normalized correlation for different angles. The solid lines are the actual correlation calculated from the DNS data, whereas the dashed-dotted lines correspond to the model (4).

assuming that the hot-wires will be less likely to have lengths greater than 100 viscous units (Hutchins et al. [2009] present data for wires where $l^{+}>100$ to demonstrate an extreme case of spatial attenuation, however such sensors are not typically deployed). Note that, $\lambda_{\theta}^{+} \approx 26$ for $\theta=90^{\circ}$ (the spanwise direction) and $\approx 200$ for $0^{\circ}$ (the streamwise direction); whereas the near-wall streak spacing (in $y$ ) is $\approx 100$ viscous units and has a length of $\approx 1000$ viscous units (in $x$ ). Hence, $\lambda_{\theta}$ is proportional to the scales of the flow.

Substituting the model for correlation (4) along with (5) into (3) leads to,

$$
\frac{\tilde{u}_{r m s}^{2}}{u_{r m s}^{2}}=\frac{2}{l^{2}} \int_{0}^{l} \mathrm{e}^{-\left(r / \lambda_{\theta}\right)^{p}} \cdot(l-r) \mathrm{d} r,
$$

where the integral can be evaluated by substituting $\left(r / \lambda_{\theta}\right)^{p}$ by another variable say, $t$. The resulting form is,

$$
\frac{\tilde{u}_{r m s}^{2}}{u_{r m s}^{2}}=\frac{2 \lambda_{\theta}}{l p}\left[\gamma\left(\frac{1}{p},\left(\frac{l}{\lambda_{\theta}}\right)^{p}\right)-\left(\frac{\lambda_{\theta}}{l}\right) \gamma\left(\frac{2}{p},\left(\frac{l}{\lambda_{\theta}}\right)^{p}\right)\right]
$$

where, $\gamma(a, x)=\int_{0}^{x} \mathrm{e}^{-t} t^{a-1} \mathrm{~d} t$, is the incomplete Gamma function [e.g., Abramowitz and Stegun, 1964, p. 260]), and $\lambda_{\theta}(\theta)$ corresponds to the distribution presented in figure 4. Note that at the two extremes of $p$ the above expression simplifies, such as, for $p=2$ (corresponding to $\theta=90^{\circ}$ ), the above equation reduces to,

$$
\frac{\tilde{u}_{r m s}^{2}}{u_{r m s}^{2}}=\frac{\lambda_{90^{\circ}}^{2}}{l^{2}}\left[l \frac{\sqrt{\pi}}{\lambda_{90^{\circ}}} \operatorname{erf}\left(\frac{l}{\lambda_{90^{\circ}}}\right)+\mathrm{e}^{-l^{2} / \lambda_{90^{\circ}}^{2}}-1\right],
$$

where, $\operatorname{erf}(x)=1 /(2 \pi) \int_{0}^{x} \mathrm{e}^{s^{2}} \mathrm{~d} s$ is the standard error function, whereas for $p=1$ corresponding to $\theta=0^{\circ},(7)$ reduces to,

$$
\frac{\tilde{u}_{r m s}^{2}}{u_{r m s}^{2}}=\frac{2 \lambda_{0^{\circ}}}{l}\left[1-\frac{\lambda_{0^{\circ}}}{l}+\frac{\lambda_{0^{\circ}}}{l} \mathrm{e}^{-\left(\frac{l}{\lambda_{0^{\circ}}}\right)}\right] .
$$

The estimate from (7) is compared to that obtained from DNS (symbols) in figure 2 with lines for the inclined wire, and the agreement is good. However, note a small difference at smaller $l^{+}$, which is an effect of the fitting function. 


\subsection{The $\vee$-probe}

Simulation of the $\vee$-probe follows the usual procedure employed for the single wire: (i) very large $l / d$ is assumed, (where, $l$ and $d$ are length and diameter of the wire) such that the temperature can be considered to be uniform over the wire length, and consequently a box-filter. (ii) No effect of the temporal response of the hot-wire is considered, i.e, a flat frequency response is assumed. (iii) For each wire, locally the velocity on the homogeneous plane is decomposed into normal $\left(u_{\perp}\right)$ and parallel $\left(u_{\|}\right)$components w.r.t the wire. The effective cooling velocity for each wire is calculated as,

$$
u_{\text {eff }}^{2}=u_{\perp}^{2}+k^{2} u_{\|}^{2} .
$$

The usual value of $k=0.2$ is assumed however its effect on $u_{r m s}^{2}$ is found to be negligible and on $v_{r m s}^{2}$ to be not appreciable, and therefore $k$ does not change the overall trends with $\theta, l$ and $\Delta s_{y}$. Hence all the calculations will be shown with $k$ set equal to zero (unless otherwise mentioned). Nevertheless, some calculations will be presented to illustrate the not-so-significant effect of $k$. (iv) As briefly mentioned above, a linear box-filter is used to calculate the $u_{\text {eff }}$ for each wire, validated against experiments in Philip et al. [2013] (where $l^{+}$is not too large). The biggest advantage of employing the linear filter is that expressions such as (1) - (3) can be derived and progress can be made in understanding the filtering effect.

Once $u_{\text {eff } 1}$ and $u_{\text {eff2 } 2}$ corresponding to wire 1 and 2 is calculated following the usual procedure of cross-wire calculations, $v$ is assumed to be much smaller than $u$ and eqn (10) is linearized such that,

$$
u_{\mathrm{eff} 1} \approx a_{1} s_{1 y} u-b_{1} s_{1 x} v,
$$

where, $a_{1}:=\sqrt{1+\left(k s_{1 x} / s_{1 y}\right)^{2}}, b_{1}:=\left(1-k^{2}\right) / a_{1}$, and $\left(s_{1 x}, s_{1 y}\right)$ are components of the unit vector along the wire. A similar equation for the second wire can also be written. These two linear equations are then inverted to obtain $u$ and $v$ [also see, Perry, 1982, Moin and Spalart, 1987].

From hereon, while considering $\vee$ (as well as $\times$ ) probes, we would like to discriminate the effects of $\Delta s$ from that of $l$ and $\theta$. To this end, we shall employ variables with overline, (e.g., $\bar{u}_{r m s}^{2+}$ ), to indicate the final measured quantities (i.e., including the effects of $\Delta s, l$ and $\theta$ ), whereas, tilde, (e.g., $\tilde{u}_{r m s}^{2+}$ ) is used to indicate only the combined effect of $l$ and $\theta$ (i.e., filtered along with wire-length).

Figure 5 shows the averaged streamwise and spanwise variances $\bar{u}_{r m s}^{2+}$ on the top row and $\bar{v}_{r m s}^{2+}$ on the bottom row, respectively, for three different $\theta$ and $\Delta s_{y}^{+}$. To begin with, consider figures $5(\mathrm{a})$ and (b) corresponding to $\Delta s_{y}^{+}=0$. This is an impractical scenario. However, is is a useful and ideal case to study the effect of $l^{+}$and $\theta$ without the complications of $\Delta s_{y}^{+}$. Furthermore, this will also be the limiting case for the $\times$-probe when $\Delta s_{z}^{+}=0$. Note that for $\Delta s_{y}^{+}=0$ (in figure 5a), $\bar{u}_{r m s}^{2+}$ decreases with increasing $\theta$. This is the same as for the inclined wire, studied in the previous section. The reason for this is that the correlations fall off quickly along the spanwise direction $\left(\theta=90^{\circ}\right)$ 

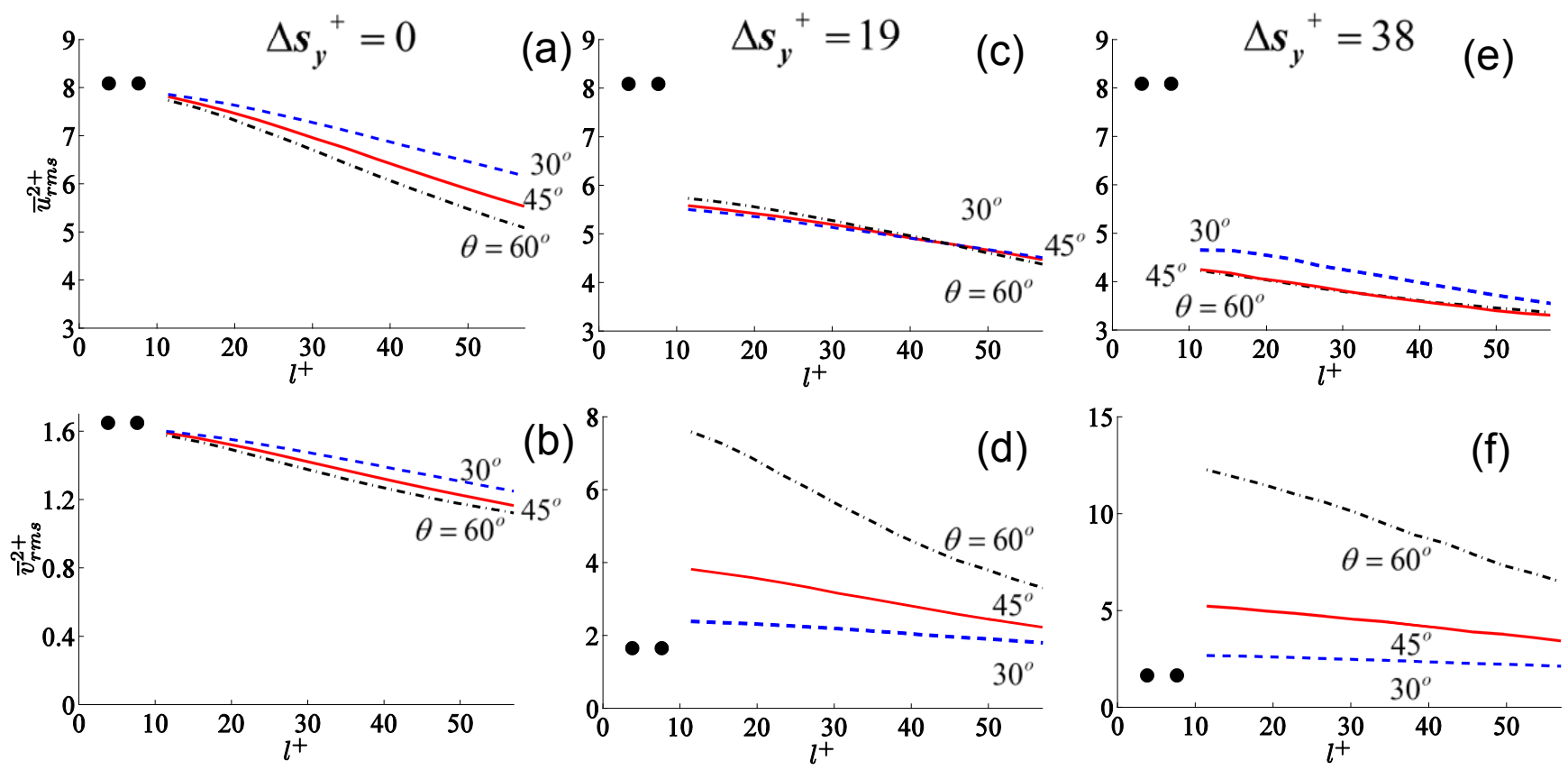

Figure 5. DNS simulation of $\mathrm{V}$-probes for the three parameters, namely, wire-length, $l^{+}$, inclination angle, $\theta$, and spacing between the wire centres, $\Delta s_{y}^{+}$, at $z^{+}=15$. Top row: distribution of $u_{r m s}^{2+}$, and bottom row: distribution of $v_{r m s}^{2+}$. (a,b) $\Delta s_{y}^{+}=0$. (c,d) $\Delta s_{y}^{+}=19$. (e,f) $\Delta s_{y}^{+}=38$. Note that unlike the top row, the ordinates for figures in the bottom row are not same.

while they decay much more slowly in the streamwise direction $\left(\theta=0^{\circ}\right)$ causing, in turn, decreasing attenuation as $\theta$ is decreased.

The effect of increasing $\Delta s_{y}^{+}$on $\bar{u}_{r m s}^{2+}$ can be observed from figures $5(\mathrm{a})$, (c) and (e) (the top row). With increasing $\Delta s_{y}^{+}$the attenuation increases. However, notice that with increasing $\Delta s_{y}^{+}$, attenuation with $l^{+}$becomes less pronounced; for instance, for $\Delta s_{y}^{+}=0$, the lines in figure 5(a) drop faster compared to $\Delta s_{y}^{+}=19$ or 38, and the same holds for $\Delta s_{y}^{+}=19$ when compared to 38. The variation of $\theta$ is more or less similar to what has been observed for $\Delta s_{y}^{+}=0$, i.e., $\theta$ close to $90^{\circ}$ is generally more attenuated than that close to $0^{\circ}$, and this is understandably the effect of the correlation function as discussed in the previous paragraph. The variation of $\bar{v}_{r m s}^{2+}$ in the bottom row shows that with increasing $\Delta s_{y}^{+}$(for a fixed $l^{+}$) the magnitude of $\bar{v}_{r m s}^{2+}$ increases at an astonishing rate and for all $\theta$. Note that the ordinates are different for all the three bottom figures to accommodate the variation of $\bar{v}_{r m s}^{2+}$ that can reach even an order of magnitude larger than the DNS ones (presented by the two black symbols). Furthermore, for a given $\Delta s_{y}^{+}$, say 19 (the middle figure in the bottom row), the errors seem to diminish with increasing $l^{+}$. The trend of $\bar{v}_{r m s}^{2+}$ with $\theta$ is understandable; the lower the $\theta$, the closer are the measured variations in $\bar{v}_{r m s}^{2+}$ to the DNS, as expected due to the above mentioned effects of the correlations. Note that even though the trends with $\theta$ in figure 5(b) compared to figures 5(d) and (f) seem reversed, the deviation from DNS (or the error) has not reversed trends, i.e., larger $\theta$ produces larger errors. 

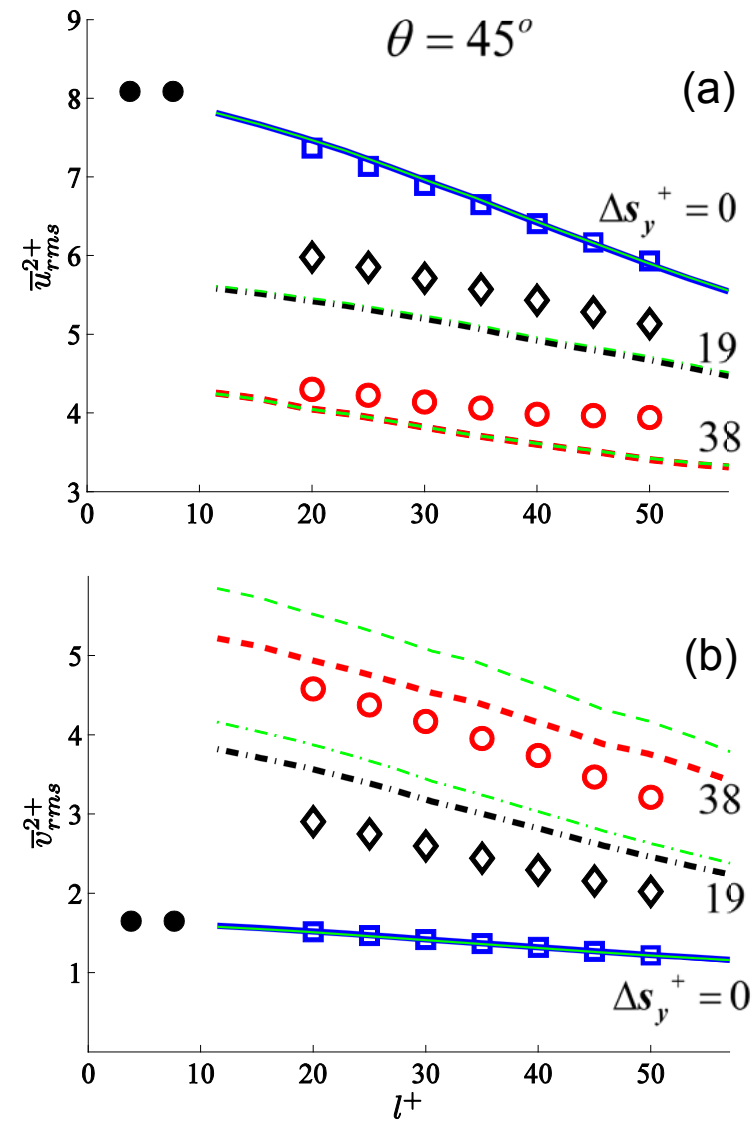

Figure 6. Comparison of DNS simulations and the model of the $\vee$-probes for $\theta=45^{\circ}$, with varying wire-lengths, $l^{+}$, and spacing between the wire centres, $\Delta s_{y}^{+}$, at $z^{+}=15$. (a) $u_{r m s}^{2+}$. (b) $v_{r m s}^{2+}$. The thick lines are DNS simulation results with $k=0$; thin (green) lines are simulations with $k=0.2$; symbols correspond to the estimations from equations (15) and (16).

However, the change in the sign of the error while moving from $\Delta s_{y}^{+}=0$ to finite values is because of the 'wire-separation effect' that now comes into play and works in a sense opposite to the 'wire-length effect' (as explained further in reference to equation 16).

To better understand these effects, a single inclination angle is isolated. Since $\theta=45^{\circ}$ is the most common configuration and also because we understand the variation with $\theta$ better, figures $6(\mathrm{a})$ and (b) show distributions of $\bar{u}_{r m s}^{2+}$ and $\bar{v}_{r m s}^{2+}$, respectively, for $\theta=45^{\circ}$ using thick lines. The thin (green) lines show the same calculations with $k=0.2$. There is no noticeable effect of $k$ on $\bar{u}_{r m s}^{2+}$, whereas the errors increase for $\bar{v}_{r m s}^{2+}$, however qualitatively the results do not change. Therefore we shall proceed to discuss the results assuming $k=0$, which will make the analysis presented below much simpler.

Consider figure $7(\mathrm{a})$ which shows a schematic of the $\vee$-probe with incoming velocity $\mathbf{u}$, and figure $7(\mathrm{~b})$ where the velocities are averaged over wires 1 and 2 . The corresponding wire-filtered velocities are shown by $\tilde{\mathbf{u}}_{1}$ and $\tilde{\mathbf{u}}_{2}$. The relationship between $u$ and $\tilde{u}$ has already been derived in (7) and depends on the correlation along each wire. So the remaining analysis can be reduced to the one shown in figure $7(\mathrm{~b})$, where only 

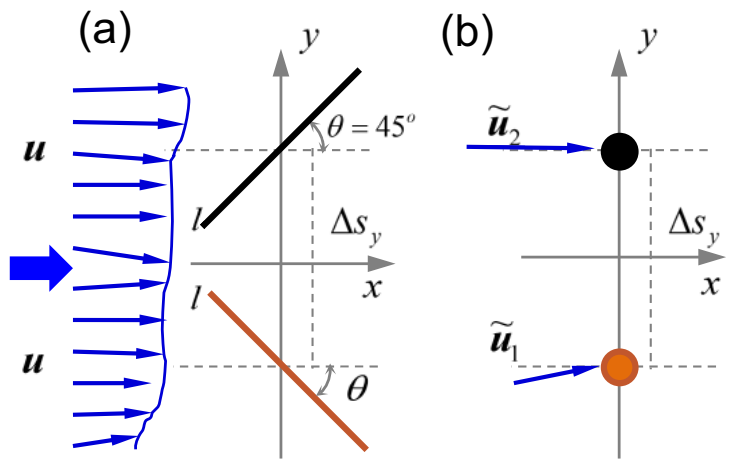

Figure 7. Schematic of the $\vee$-probe explaining the steps in the averaging process. (a) Initial configuration with an incoming velocity $\mathbf{u}$ and wire-length $l$. (b) After the velocity is averaged over the wires, denoted by $\tilde{\mathbf{u}}$, and wires have been replaced by points. The final measured velocities $(\bar{u}$ and $\bar{v})$ are only a function of $\tilde{\mathbf{u}}_{\mathbf{1}}$ and $\tilde{\mathbf{u}}_{\mathbf{2}}$, given by equation (14).

the wire-filtered velocities are shown. We consider $k=0$ and $\theta=45^{\circ}$ for simplicity, and for this case the measured instantaneous velocities can be written as:

$$
\bar{u}=\frac{\tilde{u}_{\mathrm{eff} 1}+\tilde{u}_{\mathrm{eff} 2}}{\sqrt{2}}, \bar{v}=\frac{\tilde{u}_{\mathrm{eff} 1}-\tilde{u}_{\mathrm{eff} 2}}{\sqrt{2}},
$$

where, $\tilde{u}_{\text {eff1 }}$ and $\tilde{u}_{\text {eff2 }}$ are given by (10) for each wire after the velocities are averaged over the respective wire-lengths. Note that with $k=0, u_{\text {eff }}=u_{\perp}$, and substituting the expression for $u_{\perp}$ in terms of streamwise and spanwise velocities $(u, v)$ for wires 1 and 2 , results in:

$$
\bar{u}=\frac{\tilde{u}_{1}+\tilde{u}_{2}}{2}+\frac{\tilde{v}_{1}-\tilde{v}_{2}}{2} ; \bar{v}=\frac{\tilde{u}_{1}-\tilde{u}_{2}}{2}+\frac{\tilde{v}_{1}+\tilde{v}_{2}}{2} .
$$

Decomposing the instantaneous velocity $(u)$ into mean $(U)$ and fluctuating parts $\left(u^{\prime}\right)$, $u=U+u^{\prime}$, leads to,

$$
\overline{u^{\prime}}=\frac{\tilde{u}_{1}^{\prime}+\tilde{u}_{2}^{\prime}}{2}+\frac{\tilde{v}_{1}^{\prime}-\tilde{v}_{2}^{\prime}}{2} ; \overline{v^{\prime}}=\frac{\tilde{u}_{1}^{\prime}-\tilde{u}_{2}^{\prime}}{2}+\frac{\tilde{v}_{1}^{\prime}+\tilde{v}_{2}^{\prime}}{2} .
$$

A similar expression for the mean components can also be written.

Squaring the above equations, taking the ensemble average and substituting the right hand side of (7) as $\mathrm{F}\left(l / \lambda_{45^{\circ}}\right)$ (where, $\lambda_{45^{\circ}}$ is the value of $\lambda$ at $\theta=45^{\circ}$, such that, $\left.\mathrm{F}_{u}\left(l / \lambda_{45^{\circ}}\right):=\tilde{u}_{r m s}^{2} / u_{r m s}^{2}\right)$, results in (see Appendix for the details of the derivation):

$$
\frac{\bar{u}_{r m s}^{2}}{u_{r m s}^{2}} \approx \underbrace{\left[1-0.5\left(1-\tilde{R}_{N_{u u}}\left(\Delta s_{y}\right)\right)\right]}_{\Delta s_{y} \text { effect } \Downarrow} \cdot \underbrace{\mathrm{F}_{u}\left(\frac{l}{\lambda_{45^{\circ}}}\right)}_{l \text { effect } \Downarrow},
$$

and,

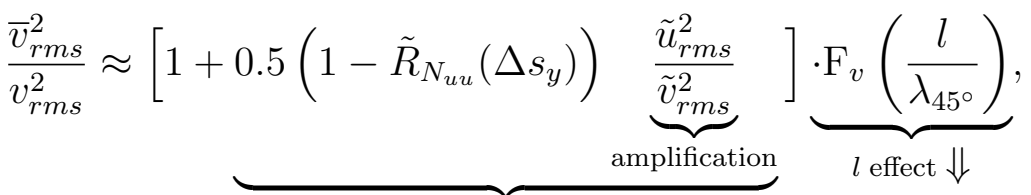

$$
\begin{aligned}
& \Delta s_{y} \text { effect } \Uparrow
\end{aligned}
$$




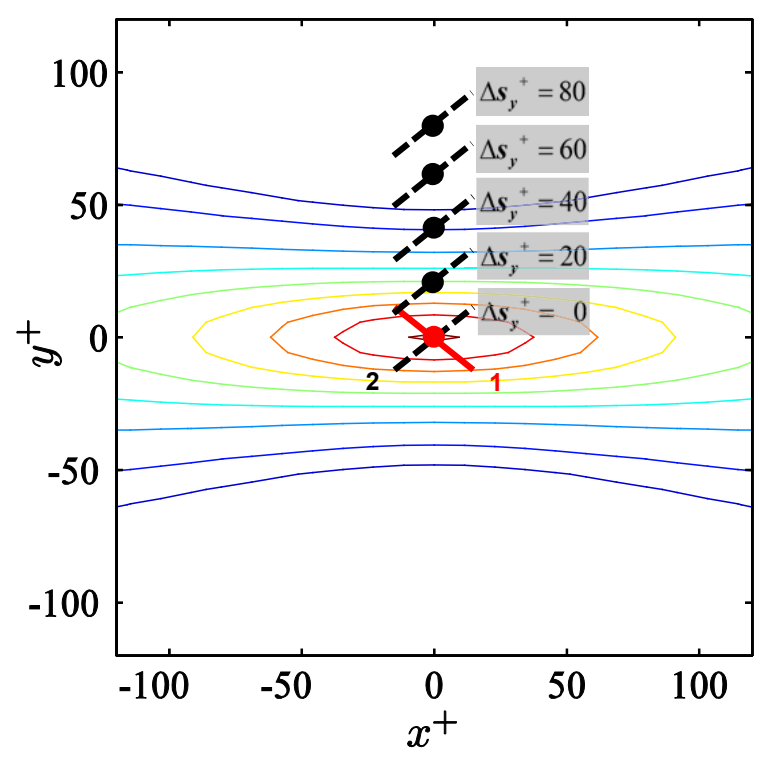

Figure 8. Contours of $R_{11}$ ( or $R_{u u}$ ) and overlayed $\vee$-probe schematic showing different $\Delta s_{y}^{+}$locations of the second wire. The magnitude of $R_{11}$ between wire-midpoints dictate the attenuation (after averaging over the wire-length is taken care of).

where, in the above expressions it is assumed that $\tilde{v}_{r m s}^{2}$ is much smaller than $\tilde{u}_{r m s}^{2}$ [see also, Suzuki and Kasagi, 1992, and the Appendix for the complete expression without this assumption, and comments on the various assumptions]. Furthermore, $\tilde{R}_{N_{u u}}\left(\Delta s_{y}\right)$ is the normalised correlation defined as,

$$
\tilde{R}_{N_{u u}}\left(\Delta s_{y}\right):=\left\langle\tilde{u}_{W 1}^{\prime}(s) \tilde{u}_{W 2}^{\prime}\left(\Delta s_{y}+s\right)\right\rangle / \tilde{u}_{r m s}^{2},
$$

where ${\tilde{u^{\prime}}}_{W 1}$ and $\tilde{u}_{W 2}^{\prime}$ are the fluctuating velocities that are averaged over the wire-length of wire- 1 and wire-2, respectively, located at a distance $\Delta s_{y}$ apart. Therefore, strictly, $\tilde{R}_{N_{u u}}$ is a function of $\left(\Delta s_{y} ; l / \lambda_{45^{\circ}}\right)$ and not just $\left(\Delta s_{y}\right)$. The angled brackets \langle\rangle are the symbol for ensemble-averaging. $\tilde{R}_{N_{u u}}$ can be obtained directly from $R_{N_{u u}}$ using eqn (2).

To illustrate the calculation of $\tilde{R}_{N_{u u}}\left(\Delta s_{y}\right)$ and clarify the mechanism of averaging in a $\vee$-probe, consider figure 8. It shows the two-point correlation of $u, R_{u u}(\mathbf{r})=$ $\left\langle u^{\prime}(\mathbf{s}) u^{\prime}(\mathbf{r}+\mathbf{s})\right\rangle$, on the plane $z^{+}=15$ where $\mathbf{r}$ is the two-dimensional position vector in the $x-y$ plane. Also shown in the figure is the schematic of two wires indicated respectively as wire 1 (solid red line) and wire 2 (broken black line) each of length, say, $l^{+}=30$, with their centre position shown with a solid dot. Wire 2 is depicted at various spanwise locations, $\Delta s_{y}^{+}$relative to wire 1 , from $\Delta s_{y}^{+}=0$ to 80 , in steps of 20 . The measured velocity $(\bar{u}, \bar{v})$, differs from the 'point' velocity, $(u, v)$ for two reasons. First, the velocity is averaged over the finite wire-length $(l)$; and second, the wire-centres are located at different positions $\left(\Delta s_{y}\right)$ in space. The case with $\Delta s_{y}^{+}=0$ shown in figure 8 isolates the effect of $l^{+}$, whereas increasing $\Delta s_{y}^{+}$introduces the effect of wire-separation. For a fixed $\Delta s_{y}$ and $l$, the original velocity field, $(u, v)$ is averaged over the wire length to give $(\tilde{u}, \tilde{v})$, which after taking into account the geometric configuration of the wires produces $(\bar{u}, \bar{v})$. During the first step of wire-length averaging, the correlation 'along 


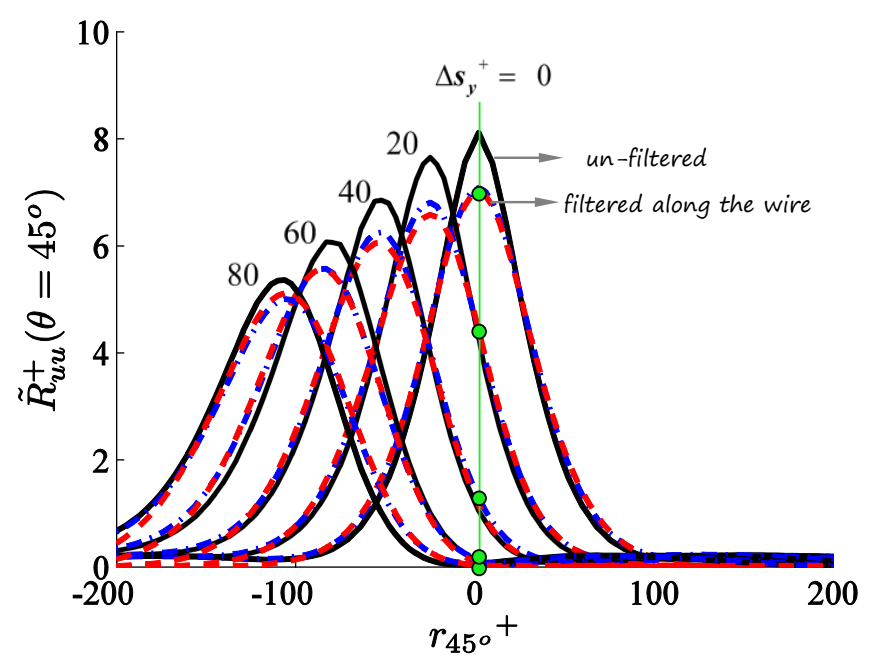

Figure 9. Streamwise correlation at $\theta=45^{\circ}$ at various $\Delta s_{y}^{+}$-locations, at $z^{+}=15$. The full (black) lines are un-averaged correlations $\left(R_{u u}\right)$ taken directly from figure 8 at the different locations of wire-2, i.e., different $\Delta s_{y}^{+}$. The broken lines are correlations averaged over the wire-length, $\tilde{R}_{u u}^{+}$, obtained by convolving the $R_{u u}$ in full (black) lines by a 'triangle' function (c.f., equation (2)), with $l^{+}=30$. Dashed-dotted lines (blue) are from the DNS and the dashed (red) lines are from the model in (4).

the wire' also changes, which is given by equation (2). This correlation, $\tilde{R}_{u u}$, is shown in figure 9 for a wire of $l^{+}=30$. Note that the DNS agrees with the model quite well. The correlations are presented along the $45^{\circ}$ lines located at $\Delta s_{y}^{+}=0$ to 80 , in steps of 20 , corresponding to the locations of wire- 2 in figure 8 . Also shown is the un-filtered correlations in full (black) lines, which are the same as those presented in contour form in figure 8. Note that the correlations are not normalised (by its value at the origin) to show the actual attenuation in $u_{r m s}^{+}$.

Now, finding the correlation between the two wires separated by a fixed distance, $\Delta s_{y}^{+}$, amounts to finding the two points in figure 9 at the origin on the abscissa, where the vertical line (shown by a full, green line) cuts $\Delta s_{y}^{+}=0$ (wire-1) and the required $\Delta s_{y}^{+}$(wire-2). For example, if wire-2 is at $\Delta s_{y}^{+}=20$, the relative correlation will be that between the top two points on the vertical line. This correlation can be normalised with the value at wire-1, (i.e. $\left.\Delta s_{y}^{+}=0\right)$ and is denoted by $\tilde{R}_{N_{u u}}\left(\Delta s_{y}^{+}\right)$. Note that the direction of $\Delta s_{y}^{+}$is the same as $r_{90^{\circ}}^{+}$.

The distribution of $\tilde{R}_{N_{u u}}\left(\Delta s_{y}^{+}\right)$obtained from figure 9 (along the solid, green line) is normalised (with the value at $\Delta s_{y}^{+}=0$ ) and plotted in figure 10, indicated with $l^{+}=30$ (recall that figure 9 is for wires with $l^{+}=30$ ). Figure 10 also shows the distribution for $l^{+}=0$ (corresponding to wire length of zero, while still separated in space) and also for $l^{+}=20,30$ and 50. Note that once a $2 \mathrm{D}$ map of $\tilde{R}_{N_{u u}}(x, y)$ is obtained (from eqn (2)) for a specific wire-length and angle, $\tilde{R}_{N_{u u}}\left(\Delta s_{y}^{+}\right)$is simply $\tilde{R}_{N_{u u}}(x=0, y)$.

From figure 10 it can be observed that compared to $l^{+}=0$, longer wires are better correlated in space; this is intuitive from figure 8 because longer wires have a better chance of measuring the same velocity than shorter wires, if separated by the same $\Delta s_{y}^{+}$. 


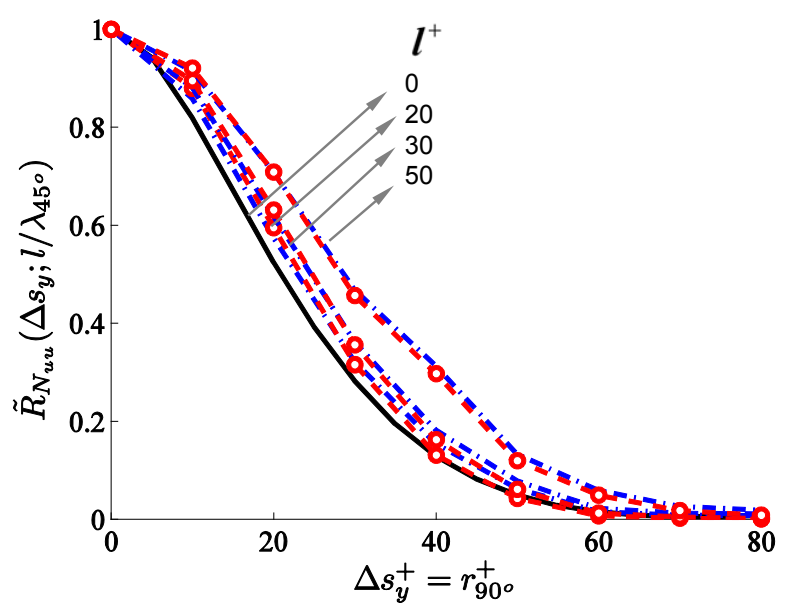

Figure 10. Correlation of wire-length averaged velocities, $\tilde{\widetilde{R}}_{N_{u u}}$ with $\Delta s_{y}^{+}$for different wire-lengths. Note that for $l^{+}=30$, the distribution of $\tilde{\tilde{R}}_{N_{u u}}$ is obtained from figure 9 .

Note, it has interesting implications for the measurements of the two-point correlation with hot-wires of finite length. The measured normalised correlation will always have a higher value than the actual one. In fact, equation (2) can be inverted, such that the actual two-point correlation can be obtained from the filtered one.

We are now in a position to explain the trends in $\bar{u}_{r m s}^{2+}$ and $\bar{v}_{r m s}^{2+}$ presented in figure 6 for the two wires at $\theta=45^{\circ} \mathrm{using}(15)$ and (16). First, consider figure 6(a) and equation (15) for $\bar{u}_{r m s}^{2+}$. Equation (15) has two parts, which can broadly be described as the effects due to the wire length $l$ and the wire-separation $\Delta s_{y}$ as indicated. The $l$-effect is well understood by the function $F_{u}$ which is given by (3) and is a strictly decreasing function. The averaging always reduces the variance, and as seen in figure 6(a) with increasing $l^{+}, \bar{u}_{r m s}^{2+}$ indeed decreases. The second effect due to $\Delta s_{y}$ is contained in the correlation $\tilde{R}_{N_{u u}}$ discussed in reference to figure 10 . As mentioned above $\tilde{R}_{N_{u u}}$ depends on $l^{+}$also; however, as seen from figure 10, the dependence is minimal. In (15), the function in square parenthesis, [ ], is also a decreasing function with $\Delta s_{y}$. This is reflected in the decrease of $\bar{u}_{r m s}^{2+}$ in figure 6 with $\Delta s_{y}^{+}$for a constant $l^{+}$. Also note that with increasing $\Delta s_{y}^{+}$, the effect of $l^{+}$diminishes, (e.g., distribution with $\Delta s_{y}^{+}=0$ has a sharper slope than that from $\left.\Delta s_{y}^{+}=38\right)$. This is because with increasing $l^{+}$the two wires are better correlated, as seen in figure 10.

The overall effect on $\bar{u}_{r m s}^{2+}$ can be simply observed by noticing the first expression in (14) for $\bar{u}$. Since $\tilde{v}_{1,2}$ are much smaller than their $\tilde{u}$ counterpart, neglecting the second term shows that $\bar{u}$ is simply the average of $\tilde{u}$ obtained from two wires. Furthermore, since $\tilde{u}$ decreases with $l^{+}$and averaging reduces the variance, the variance of $\bar{u}, \bar{u}_{r m s}^{2+}$ will always reduce. How much it reduces is approximately given by (15).

The distribution in $\bar{v}_{r m s}^{2+}$ in figure 6 (b) can be explained by equation (16). Similar to (15), here too the contribution to $\bar{v}_{r m s}^{2+}$ can be approximately written as a product of the effects of $l^{+}$and $\Delta s_{y}^{+}$. The $l^{+}$effect given by $\mathrm{F}_{v}$ is a relation of the form (7) presented 
for $u$-velocity. This will again depend on the $v$-correlations, which are not shown here. However, the effect is similar to that of $\mathrm{F}_{u}$; it decreases with increasing $l^{+}$. The effect of $\Delta s_{y}^{+}$reverses the role here. The function $\tilde{R}_{N_{u u}}$ is a decreasing function which makes the quantity in the square parenthesis, [ ], an increasing function with $\Delta s_{y}^{+}$, involving another term $\tilde{u}_{r m s}^{2+} / \tilde{v}_{r m s}^{2+}$ which is always much greater than one and 'amplifies' the effect of $\Delta s_{y}^{+}$. In other words, both $l^{+}$and $\Delta s_{y}^{+}$have opposite effects on $\bar{v}_{r m s}^{2+}$. In figure 6(b), for $\Delta s_{y}^{+}=0$, because of the $l$-effect, $\bar{v}_{r m s}^{2+}$ decreases, however as soon as $\Delta s_{y}^{+} \neq 0, \bar{v}_{r m s}^{2+}$ increases beyond the DNS values (shown as two thick black dots). The effect of $l^{+}$is as expected, increased $l^{+}$leads to reduced $\bar{v}_{r m s}^{2+}$.

In other words, the second equation in (14) for $\bar{v}$ shows (again neglecting the smaller $\left.\tilde{v}_{1,2}\right)$ that it is a 'difference' of $\tilde{u}$ at wire- 1 and 2 , which is a result of the way velocities are obtained from cross-wires. Both $\tilde{u}_{1,2}$ are relatively large in magnitude compared to their $v$-counterparts: a small mismatch in the two big numbers $\left(\tilde{u}_{1}\right.$ and $\left.\tilde{u}_{2}\right)$ results in a large difference, and consequently a larger error in the variance of $\bar{v}$, i.e, a large, $\bar{v}_{r m s}^{2+}$ as evident in figure $6(\mathrm{~b})$.

It is of interest to see if any estimation of $\bar{u}_{r m s}^{2+}$ and $\bar{v}_{r m s}^{2+}$ can be made given that we have a model for $R_{N}$ from eqn (4). Estimation of $\bar{u}_{r m s}^{2+}$ is made using eqn (15), with $\mathrm{F}_{\mathrm{u}}$ given by the right hand side of $(7)$ and $\tilde{R}_{N_{u u}}$ calculated as described in figure 10 . The results are shown in figure $6(\mathrm{a})$ with symbols for three different $\Delta s_{y}$ and varying $l^{+}$. The estimation performs reasonably well considering that only the model (4) is used in the evaluation. The equation (16) for $\bar{v}_{r m s}^{2+}$ is considerably more difficult to evaluate. The main reason is that $\mathrm{F}_{v}$ still depends on the $v$-correlation for which we have not built a model. In principle this can be done, however producing an $\mathrm{F}_{v}$ is beyond the scope of this paper. So as a first approximation we assume that $\mathrm{F}_{v}$ is the same as $\mathrm{F}_{u}$. Note that (16) involves the 'amplification factor', $\tilde{u}_{r m s}^{2+} / \tilde{v}_{r m s}^{2+}$, which can be obtained without the knowledge of $\mathrm{F}_{\mathrm{v}}$. (This is more evidently observed in equation (28 from the Appendix) which is the same as (16) written in terms of actual variances, and the amplification factor appears along with $\mathrm{F}_{u}$ rather than $\mathrm{F}_{v}$.) With the above mentioned assumptions equation (16) is evaluated and the estimated distribution of $\bar{v}_{r m s}^{2+}$ is presented in figure 6(b) using symbols consistent with figure 6(a). Note that for $\Delta s_{y}^{+}=0$ the estimation is quite close; however, for higher $\Delta s_{y}^{+}$even though the trends of the symbols and the lines match, the actual values are somewhat under-predicted. However, even with the approximations involved the essential physics of averaging is captured by equations (15) and (16), given only the model (4).

\subsection{The $\times$-probe for $u$-v-velocity (and the differences from $\vee$-probes)}

Here we investigate the averaging effects of the $\times$-probe, shown schematically in figure 1(c), for the measurements of streamwise and spanwise velocities. The $\times$-probe has two wires separated in the wall-normal direction $z$ by a distance $\Delta s_{z}$, and as in the previous section, we shall consider the velocities to be measured at $z^{+}=15$, such that the wires are placed above and below $z^{+}=15$ by $\Delta s_{z}^{+} / 2$. The calculation of $u$ and $v$ 

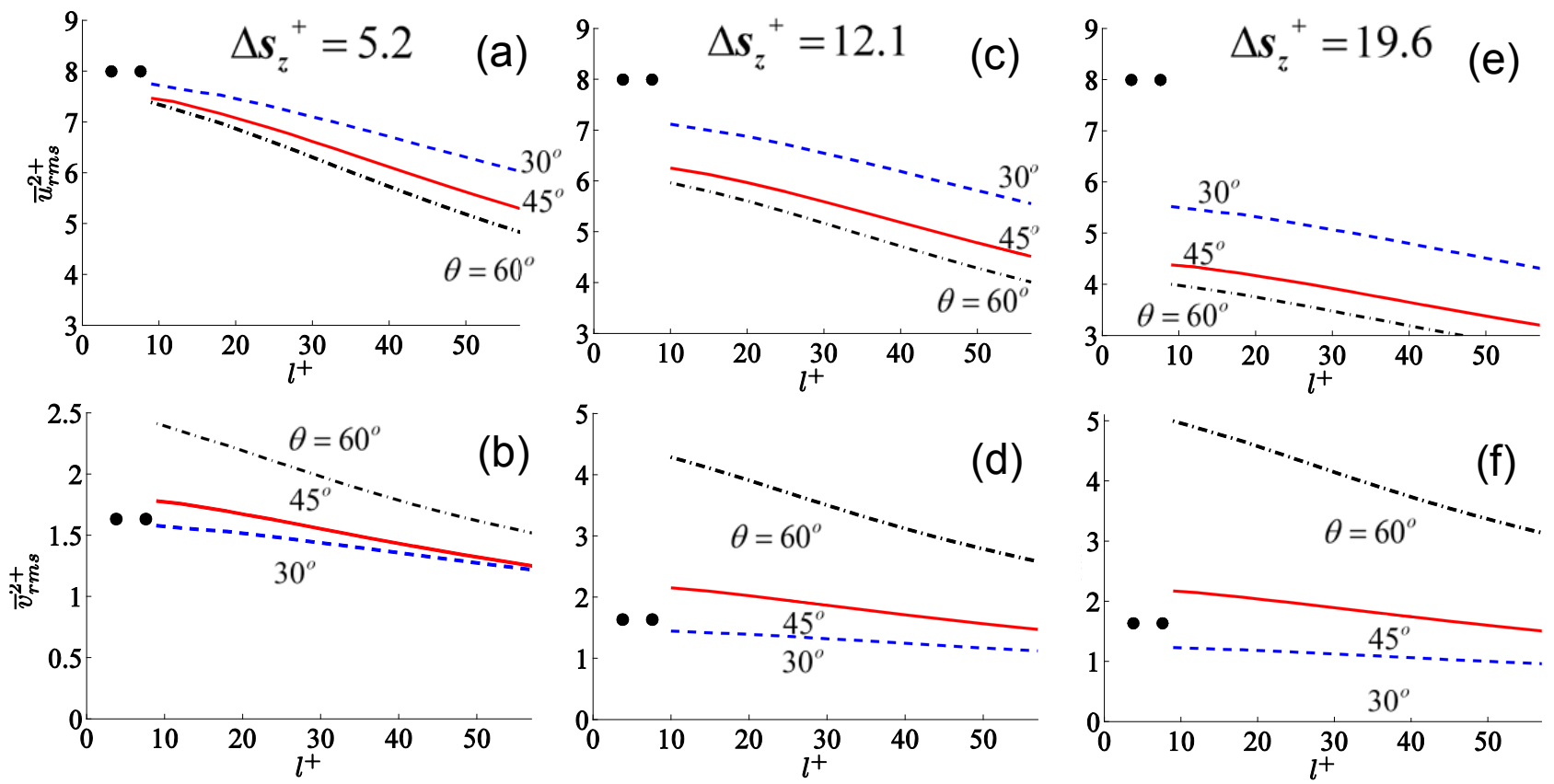

Figure 11. DNS simulation of $\times$-probes for the three parameters, namely, wirelength, $l^{+}$, inclination angle, $\theta$, and spacing between the wire centres, $\Delta s_{z}^{+}$. Top row: distribution of $u_{r m s}^{2}$, and bottom row: distribution of $v_{r m s}^{2}$. (a,b) $\Delta s_{y}^{+}=5.2$. (c,d) $\Delta s_{y}^{+}=12.1$. (e,f) $\Delta s_{y}^{+}=19.6$. Note that unlike the top row, the ordinates for figures in the bottom row are not the same.

using the DNS follows the same procedure described previously for the $\vee$-probe, again taking $k=0$. Figures $11(\mathrm{a}-\mathrm{f})$ show $\bar{u}_{r m s}^{2+}$ (top panel) and $\bar{v}_{r m s}^{2+}$ (bottom panel) at the nominal value of $z^{+}=15$ for three different $\Delta s_{z}^{+}$and angles, as indicated in the figures.

For $\Delta s_{z}^{+}=0$, the $\times$ and the $\vee$ probes (for $\Delta s_{y}^{+}=0$ ) are the same. For this reason $\Delta s_{z}^{+}=0$ is not shown in figure 11 . This result can be viewed in figures 6 (a) and (b). Note that the effect of the variation in angle is well understood; with increasing $\theta$, the error increases, which is primarily due to the correlations dropping faster along the spanwise direction than the streamwise, similar to $\vee$-probes (c.f. figure 5) . With increasing $\Delta s_{z}^{+}$, the trends look roughly similar to the $\vee$-probe with increasing $\Delta s_{y}^{+}$, however with some subtle differences. Comparing figures 11 and 5 for the $\times$-probe and $\vee$-probe, respectively, at $\Delta s_{z}^{+} \approx 19$ shows that for the same spacing between the wires, the errors in $u_{r m s}^{+}$are larger, whereas those in $v_{r m s}^{+}$are smaller for $\times$-probes compared to the $\vee$-probes. These effects can be better studied by isolating a single $\theta$, say $45^{\circ}$ and comparing with the $\vee$-probe.

Figure 12 shows $\bar{u}_{r m s}^{2+}$ (top) and $\bar{v}_{r m s}^{2+}$ (bottom) for $\theta=45^{\circ}$ at different $\Delta s_{z}^{+}$with lines. Comparing this figure with figure 6 shows immediately, as mentioned above, the larger error in $u_{r m s}^{+}$and smaller error in $v_{r m s}^{+}$for the $\times$-probe. The difference is due to the way the wires are arranged in $\times$ and $\vee$-probes about $z^{+}=15$. The wire arrangements for the two configurations experience different mean flow as well as different levels of fluctuations; both the wires in the $\vee$-probe have the same mean velocity which is not 
true for the $\times$-probe. It was mentioned in Philip et al. [2013] (in the context of a single wire) that, 'for a linear filter the filtered mean velocity is affected by the actual mean velocity, whereas the filtered fluctuations are only affected by the actual fluctuations'. This is also evident in equation (14), where the filtered fluctuating velocities are a function only of the actual fluctuating velocities at each wire. In short, this implies that the differences in the errors of $\bar{u}_{r m s}^{2+}$ and $\bar{v}_{r m s}^{2+}$ for the $\vee$ - and $\times$-probes cannot be due to the variation of mean velocity between the two wires of the $\times$-probe (which is non-existent in a $\vee$-probe). Rather this difference in $u_{r m s}^{+}$and $v_{r m s}^{+}$must be due to the differences in the fluctuating velocities at the wires. Indeed, it will be shown (later) that the $\times$-probe introduces some errors in reproducing the mean velocities while no such errors are present in $\vee$-probes, and that they are not related to the errors in variances (at least for linear filters which have been shown to be a good approximation for the hot-wire in Philip et al. [2013]).

Starting from equation (14) for the wire at $\theta=45^{\circ}$, which is valid for $\times$-probes too, expressions for the measured or filtered variances can be obtained by the Taylor expansion of variances and neglecting terms of order $\left(\Delta s_{z}\right)^{2}$ and above (details of which are given in the Appendix):

$$
\frac{\bar{u}_{r m s}^{2}}{u_{r m s}^{2}} \approx\left[1-0.5\left(1-\tilde{R}_{N_{u u}}\left(\Delta s_{z}\right)\right)\right] \cdot \mathrm{F}_{u_{0}}\left(\frac{l}{\lambda_{45^{\circ}}}\right)
$$

and,

$$
\begin{aligned}
& \frac{\bar{v}_{r m s}^{2}}{v_{r m s}^{2}} \approx-0.5 \underbrace{\left[1-\tilde{R}_{N_{v v}}\left(\Delta s_{z}\right)\right]}_{\text {significant for } \times \text {-probe }} \cdot \mathrm{F}_{v_{0}}\left(\frac{l}{\lambda_{45}}\right)+ \\
& {\left[1+0.5\left(1-\tilde{R}_{N_{u u}}\left(\Delta s_{z}\right)\right) \frac{\tilde{u}_{r m s}^{2}}{\tilde{v}_{r m s}^{2}}\right] \cdot \mathrm{F}_{v_{0}}\left(\frac{l}{\lambda_{45^{\circ}}}\right),}
\end{aligned}
$$

where, $\mathrm{F}_{u_{0}}$ is again the ratio, $\tilde{u}_{r m s}^{2} / u_{r m s}^{2}$, however evaluated at $z_{0}$, the $z$-location at the middle of the two wires, i.e., $\Delta s_{z} / 2$ from each wire (c.f. figure $\left.1(\mathrm{c})\right), \tilde{R}_{N_{u u}}\left(\Delta s_{z}\right)$ is the $u$-correlation between $\tilde{u}_{1}$ and $\tilde{u}_{2}$ located at wire 1 and 2 , respectively, separated by $\Delta s_{z}$, and similar definitions hold for the $v$ component terms. Note that (18) is the same as for the $\vee$-probe, whereas (19) has an important contribution from a new term indicated with an under-brace in (19), which was very small and neglected for $\vee$-probes. The difference in error between $\times$ and $\vee$-probes arises because of the differences in the distributions of $\tilde{R}_{N_{v v}}\left(\Delta s_{z}\right)$ and $\tilde{R}_{N_{v v}}\left(\Delta s_{y}\right)$. Note that the new term (or the first one) in (19) involving $\tilde{R}_{N_{v v}}\left(\Delta s_{z}\right)$ has a negative sign, implying that it will try to make the $\bar{v}_{r m s}^{2}$ smaller than the original whereas the second term (also present for $\vee$-probes) primarily makes $\bar{v}_{r m s}^{2}$ larger. It is this opposing effect that makes the errors for the variance of the $\times$-probe smaller than those for the $\vee$-probe (whereas there is no such significant 'error-cancellation' happening for the $\vee$-probes). It is now evident that the relative magnitudes of the correlations 'along the wire' and between the 'centre of the two wires' are the ones that control the errors in variances for both $\times$ - and $\vee$-probes.

Figure 13 shows the four correlation functions that enter into the estimation of $u$ and $v$ variances. In the present context, for both the $\times$ - and $\vee$-probe, the centres of 
both wires are at the same $x$ location; hence, the correlations at $\Delta x^{+}=0$ are of primary relevance. Figures 13(a) and (b) show $R_{N_{u u}}$ and $R_{N_{v v}}$ in the homogeneous $x$-y plane at $z^{+}=15$, along with the schematic of a $\vee$-probe where one wire (in red) is placed at the point of maximum correlation and the other (in blue) is at distance $\Delta s_{y}^{+}=12$ from wire 1. In a similar way, figures $13(\mathrm{c})$ and (d) show $R_{N_{u u}}$ and $R_{N_{v v}}$ in the $x z$-plane, where the correlations are calculated with the reference point (the point of maximum correlation) at $z^{+}=9\left(=15-\Delta s_{y}^{+} / 2\right)$. Also shown is an $\times$-probe (projected in the $x z$-plane) with the bottom (red) wire at $z^{+}=9$ with $\Delta s_{z}^{+}=12$, such that the top (blue) wire is at $z^{+}=21$, with the centre of the $\times$-probe at $z^{+}=15$, as for the $\vee$-probe in the top panel. It is clear in figure $13(\mathrm{~b})$ that $R_{N_{v v}}$ for the $\vee$-probe changes very little from one wire to the next, whereas $R_{N_{v v}}$ drops dramatically in figure $13(\mathrm{~d})$ for the $\times$-probe. This in turn makes the first term in equation (19) the relevant term for $\bar{v}_{r m s}^{2+}$ in $\times$-probes (which could be neglected for the $\vee$-probe). This term cancels the second term making the $v_{r m s}^{2+}$ error for the $\times$-probe relatively less due to the 'error-cancellation'.

Note that the form of the equations (18) and (19) are such that they can be inverted to give the actual variance, $\left(u_{r m s}^{2}\right.$ or $\left.v_{r m s}^{2}\right)$, given the 'measured' variances $\left(\bar{u}_{r m s}^{2}\right.$ or $\left.\bar{v}_{r m s}^{2}\right)$, or, vice-versa. However, to perform this calculation we need some estimates of $\tilde{R}_{N_{u u}}\left(\Delta s_{z}\right), \tilde{R}_{N_{v v}}\left(\Delta s_{z}\right), \mathrm{F}_{u_{0}}\left(l / \lambda_{u, 45^{\circ}}\right)$ and $\mathrm{F}_{v_{0}}\left(l / \lambda_{v, 45^{\circ}}\right)$, where, $\lambda_{u, 45^{\circ}}$ and $\lambda_{v, 45^{\circ}}$ are the length scales for the $u$ and $v$-correlations along $\theta=45^{\circ}$ in the $x$-y plane at $z=z_{0}$, i.e., $z^{+}=15$. To estimate $\lambda$, we employ the correlation model of the form,

$$
R_{N_{u u}}\left(r, \theta=45^{\circ}\right)=\mathrm{e}^{-\left(r / \lambda_{u, 45^{\circ}}\right)^{m} 45^{\circ}},
$$

where, $m_{45^{\circ}}=1.5-0.5 z^{+} / R e_{\tau}$. This is because we have already seen that for $\theta=45^{\circ}$ the exponent is between $\theta=0^{\circ}$ (where, $m=2$ ) and $\theta=90^{\circ}$ (where, $m=1$ ), and as we reach the outer edge of the boundary layer, or centre of the channel, $m=1$ is the best representation for a correlation (c.f., Philip et al. [2013]). A similar correlation for $R_{N_{v v}}\left(r, \theta=45^{\circ}\right)$ is also used with the same functional form of $m_{45^{\circ}}$ as for $u$-correlation. At $z^{+}=15$, the above mentioned functional form of the correlations give $\lambda_{u, 45^{\circ}}=37$ and $\lambda_{v, 45^{\circ}}=50$. Note that referring back to figures $13(\mathrm{a})$ and (b) indeed shows that $\lambda_{v, 45^{\circ}}$ greater than $\lambda_{u, 45^{\circ}}$ along the $45^{\circ}$ line where wire 1 (in red) is placed. We recall that even though for an $\times$-probe no wires are placed at $z^{+}=15$, because of the Taylor expansion all that is needed (within the approximations) is the correlation at the mid-point of the two wires, i.e., $z^{+}=15$ along the direction of the wires. Once $\lambda_{u, 45^{\circ}}$ and $\lambda_{v, 45^{\circ}}$ are known, $\mathrm{F}_{u_{0}}$ and $\mathrm{F}_{v_{0}}$ in equations (18) and (19) can be calculated using the expressions of the form (7). The estimation of $\tilde{R}_{N_{u u}}\left(\Delta s_{z}\right)$ and $\tilde{R}_{N_{v v}}\left(\Delta s_{z}\right)$ are more challenging. Ideally we should follow the steps described in detail in connection with the $\vee$-probe in the previous section, by first finding a three-dimensional correlation map around wire 1 and then convolving the positions along the two wires with a 'triangle' function to find the resultant correlation. However, as seen in the previous section, c.f., figure 10, $\tilde{R}_{N_{u u}}$ is not very different from $R_{N_{u u}}$; hence, as a first approximation we estimate $\tilde{R}_{N_{u u}}$ with $R_{N_{u u}}$ and use a similar estimation for $\tilde{R}_{N_{v v}}$. The distributions of $R_{N_{u u}}\left(\Delta s_{z}\right)$ and $R_{N_{v v}}\left(\Delta s_{z}\right)$ are obtained from figures $13(\mathrm{c})$ and (d), respectively at $\Delta x^{+}=0$. 


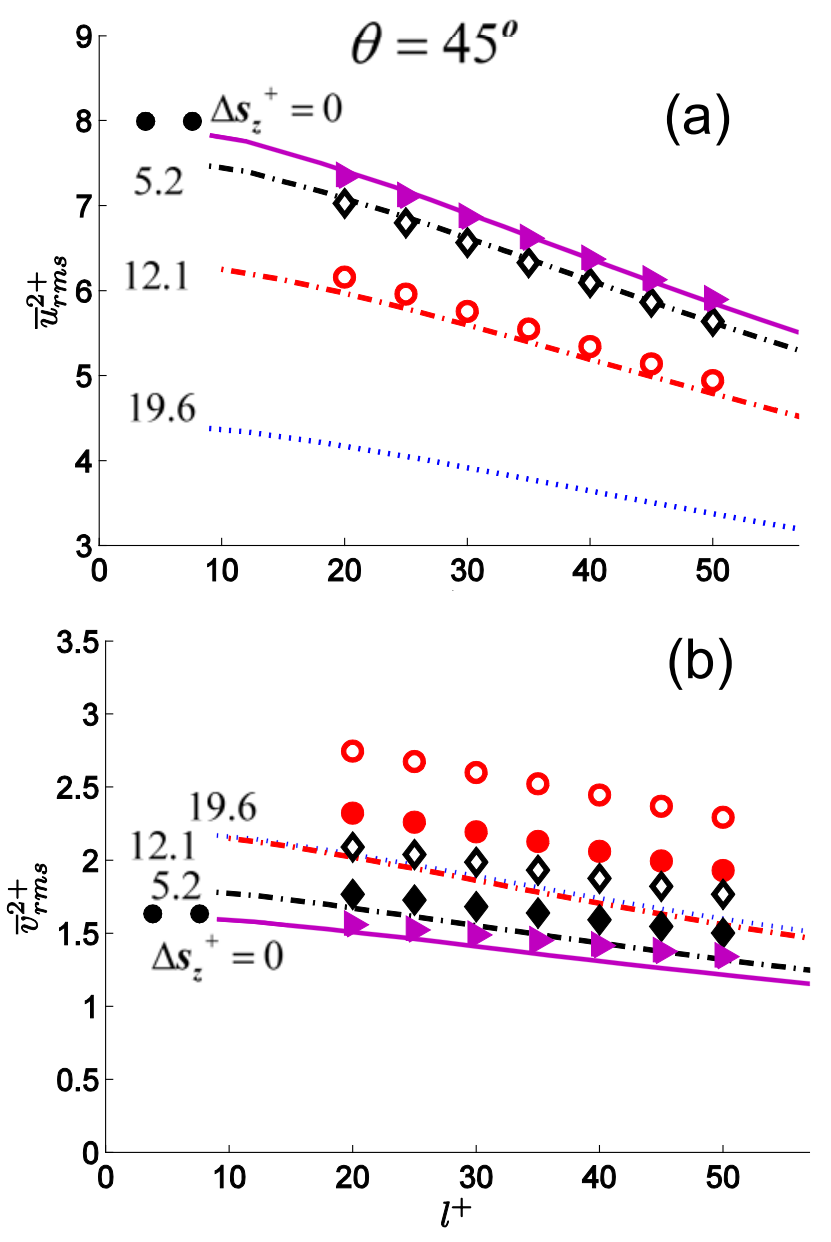

Figure 12. Comparison of DNS simulations and the model of the $\times$-probes for $\theta=45^{\circ}$, with varying wire-lengths, $l^{+}$, and spacing between the wire centres, $\Delta s_{z}^{+}$, at $z^{+}=15$. (a) $u_{r m s}^{2+}$. (b) $v_{r m s}^{2+}$. The full thick lines are DNS simulation results (with $k=0$ ). Symbols correspond to the estimations from equations (18) for $u_{r m s}^{2+}$, and (19) for $v_{r m s}^{2+}$, with 'triangles', 'diamonds' and 'circles' corresponding to $\Delta s_{z}^{+}=0,5.2,12.1$, respectively. The empty symbols in (b) correspond to only the second term in (19), whereas full symbols include both the terms in (19).

The estimates of $\bar{u}_{r m s}^{2}$ and $\bar{v}_{r m s}^{2}$ can now be evaluated from equations (18) and (19). Figure 12(a) shows, in (empty) symbols $\bar{u}_{r m s}^{2}$ evaluated from (18) for three $\Delta s_{z}^{+}$ corresponding to $\Delta s_{z}^{+}=0,5.2$ and 12.1. As mentioned previously approximation of small $\Delta s_{z}$ would not hold well beyond $\Delta s_{z}^{+} \approx 15$ and therefore, no estimation is presented in figure $12(\mathrm{a})$ for $\Delta s_{z}^{+}=19.6$. The estimation seems to work quite well. This was expected because the same estimation also gave favorable results for the $\mathrm{V}$ probe, and as expected the errors in $u_{r m s}^{2}$ increase with $\Delta s_{z}^{+}$, and the increase is more for an $\times$-probe compared to a $\vee$-probe, for $\Delta s_{y}^{+}$is the same as $\Delta s_{z}^{+}$. This is in accordance with figures 13 (c) and (a) where the $u$-correlation for the $\times$-probe drops much faster than for the $\vee$-probe. Estimations for $\bar{v}_{r m s}^{2}$ from equation (19) are shown in figure $12(\mathrm{~b})$ with 'full' symbols. Also shown in 'empty' symbols is the estimation with only the second term in (19) - which was the approximation previously used for a $\vee$-probe. For 


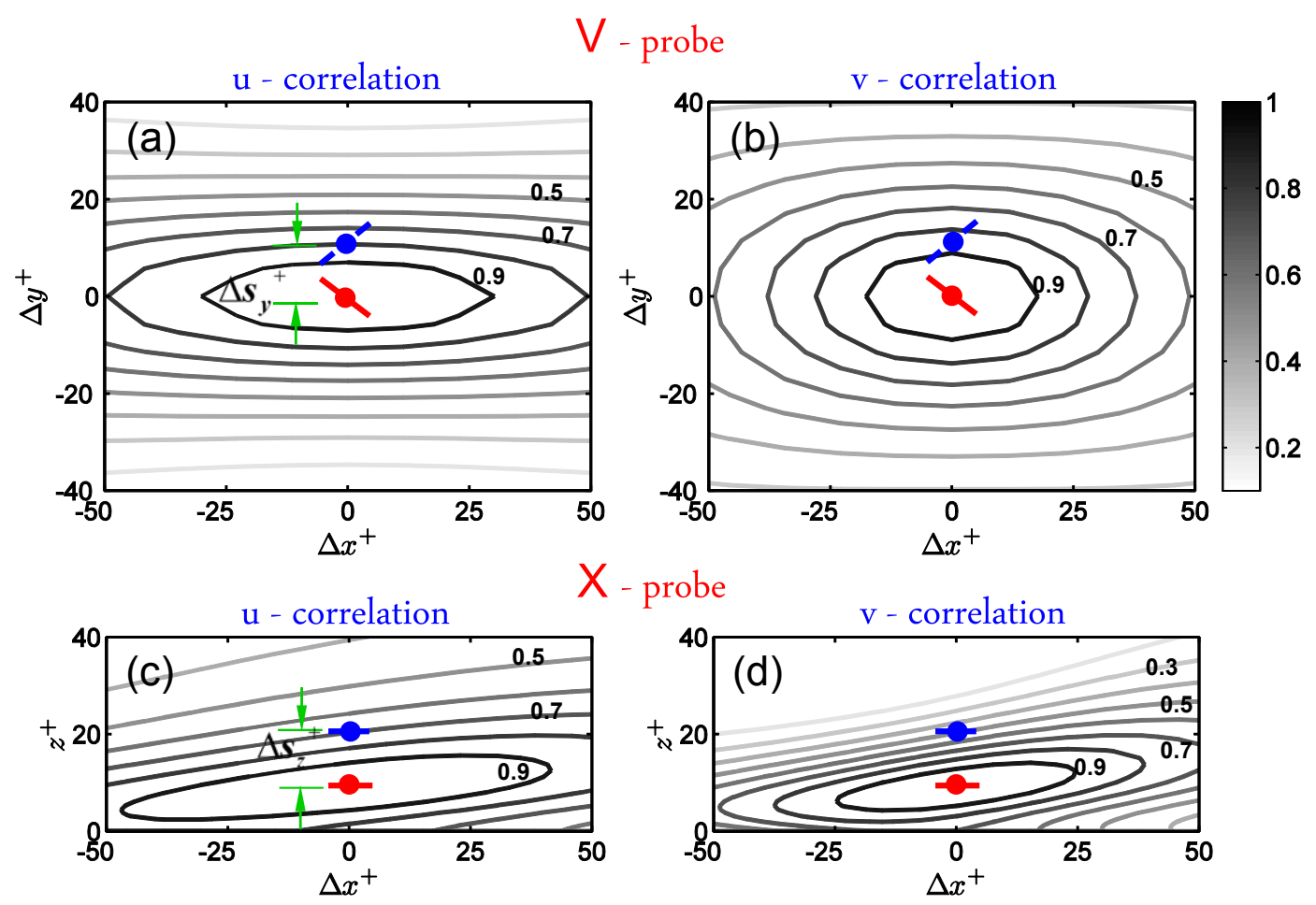

Figure 13. Two point correlations for $u, R_{u u}$ and for $v, R_{v v}$ with nominal wire positions at $z^{+} \approx 15$. Top row corresponds to the $\vee$-probe with correlations in $\Delta y^{+}$$\Delta x^{+}$plane whereas bottom row refers to the $\times$-probe with correlations in $z^{+}-\Delta x^{+}$ plane. (a) $R_{u u}$ for $\vee$-probe. (b) $R_{v v}$ for $\vee$-probe. (c) $R_{u u}$ for $\times$-probe. (d) $R_{v v}$ for $\times$-probe.

the $\times$-probes the second term in (19) gives an increase in error whereas addition of the new term has an opposite effect and the errors decrease. The new term (the first in (19)) becomes dominant in $\times$-probes because the $v$-correlation between the two wires (see figure 13(d)) drops much faster than the $v$-correlation (see figure 13(b)) between wires in a $\vee$-probe. Also see the Appendix for further details on the approximations used in arriving at equation (19).

To explain the difference between $\vee$ - and $\times$-probes in a different way, consider equation (14) for $\bar{v}$, which has contributions from the $\tilde{u}$ and $\tilde{v}$ of two wires. In the $\vee-$ probe the second term (with $\tilde{v}$ ) was negligible and the $\bar{v}$ is dominated by the 'difference of two large numbers' ( $\tilde{u})$, and consequently the variance goes up, i.e., a 'positive error'. However, in the $\times$-probe the second term in (14) (involving $\tilde{v}$ ) also becomes important, which is the contribution due to averaging. Since any averaging will only decrease the variance, the error is like a 'negative error'. Both these errors cancel each other out in the $\times$-probe to give an overall smaller error than the $\vee$-probe. 


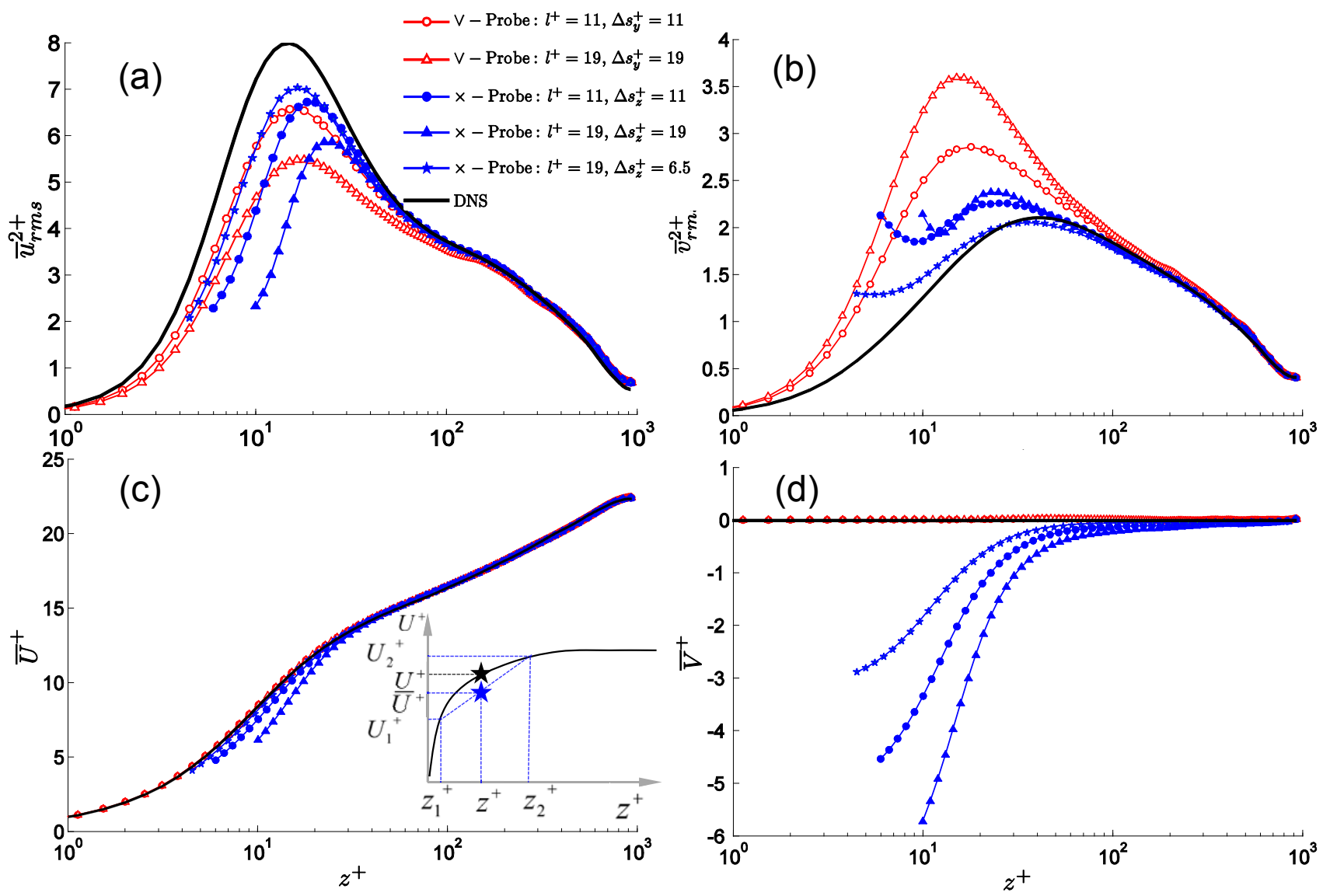

Figure 14. Variation of mean velocities and variances with $z$-locations for the $\vee$ - and $\times$-probe at $\theta=45^{\circ}$. The full (black) line is the un-averaged DNS results, whereas open and closed symbols correspond to $\vee$ - and $\times$-probes, respectively. (a) $u_{r m s}^{2}$. (b) $v_{r m s}^{2}$. (c) Mean streamwise velocity, $U$. (d) Mean spanwise velocity, $V$. The inset in (c) shown the attenuation in $U$ in the $\times$-probe.

\section{Effect of wall-normal distance $(z)$}

The effect of the wall-normal distance on the variances and the mean for both $\vee$ and $\times$ probes at $\theta=45^{\circ}$ is illustrated in figure 14. The thick (black) lines are the DNS results without any averaging, and the empty (red) and filled (blue) symbols are for the $\vee$ and $\times$-probe, respectively. Primarily two cases are considered for each wire, $l^{+}=\Delta s_{y}^{+}=11$ and 19 (for $\vee$-probe), and $l^{+}=\Delta s_{z}^{+}=11$ and 19 (for $\times$-probe). An extra case of $\times$-probe with $l^{+}=19$ and $\Delta s_{z}^{+}=6.5$ is also shown, which is a typical probe built for use in the wind tunnel at the University of Melbourne.

The effect of $l^{+}$and $\Delta s_{y}^{+}$or $\Delta s_{z}^{+}$at $z^{+}=15$ was already discussed in detail in the previous section. For the same $l^{+}$and $\Delta s^{+}$, the $\vee$-probe had less errors in $u_{r m s}^{2}$ than the $\times$-probe whereas the opposite was true in $v_{r m s}^{2}$. The plausible reason was that for $u_{r m s}^{2}$, the correlation between the two wire-centres of the $\vee$-probe was significantly higher than for the $\times$-probe, whereas in $v_{r m s}^{2}$, there was cancellation of errors in the $\times$-probe which makes the errors smaller than in the $\vee$-probe. This situation in $\tilde{v}_{r m s}^{2}$ does not change 
with $z^{+}$as observed in figure 14(b) where the $\times$-probe performs much better than the $\vee$. However, figure 14 (a) shows that close to $z^{+}=15$ the errors in $u_{r m s}^{2}$ for the $\times$-probe are higher than for the $\vee$-probe, but this situation reverses for greater $z^{+}$. A probable reason for the 'switching' of errors for $u_{r m s}^{2}$ in $\vee$ - and $\times$-probes at higher $z^{+}$, say $z^{+}=20$, is given in figure 15. Figure 15 (a) corresponds to the $\vee$-probe and shows the correlation in the $x$ - $y$ plane at $z^{+}=19.5$, whereas the bottom figure corresponds to the $\times$-probe and the correlation shown is in the $x z$-plane with the fixed point at $z^{+}=13.7$, indicating the location of wire 1 . With $\Delta s_{z}^{+}=12$, the mid-point between wires 1 and 2 is $z^{+} \approx 19.7$. Figures 15(a) and (b) for the nominal wire position at $z^{+}=20$ should be compared to figure $13(\mathrm{a})$ and (c) for $z^{+}=15$. For the $\vee$-probe, compared to $z^{+}=15$ (c.f., figure $13(\mathrm{a})$ ), at $z^{+}=20$ (c.f., figure $15(\mathrm{a})$ ) the wire-midpoints (shown by blue and red dots) are approximately equally or less correlated. This slightly increases the attenuation in the $u_{r m s}^{2}$ in $\vee$-probes at $z^{+}=20$. However, for the $\times$-probe compared to $z^{+}=15$ (c.f., figure 13(c)), at $z^{+}=20$ (c.f., figure 15(b)), the wire-midpoints are much better correlated. This significantly reduces the attenuation in the $\times$-probe at $z^{+}=20$. Thus, the reduced correlation between $\vee$-probe wires and a substantially increased correlation for the $\times$-probe wires at $z^{+}=20$ as compared to $z^{+}=15$ contributes to a smaller error in $u_{r m s}^{2}$ measured by $\times$-probes further from the wall. This increased correlation at still higher $z^{+}$is the major cause of improved $u_{r m s}^{2}$ for the $\times$-probe.

The mean velocity profiles, $U$ and $V$, corresponding to the variances in figures 15(a) and (b) are shown in figures $15(\mathrm{c})$ and (d), respectively. The $\vee$-probe produces the same mean as the DNS because the whole probe sits in a plane of zero mean shear or homogeneous plane. The mean velocities $(U, V)$ obtained from eqn (13) after using $u=U+u^{\prime}$, are given by:

$$
\bar{U}=\frac{\tilde{U}_{1}+\tilde{U}_{2}}{2}+\frac{\tilde{V}_{1}-\tilde{V}_{2}}{2} ; \bar{V}=\frac{\tilde{U}_{1}-\tilde{U}_{2}}{2}+\frac{\tilde{V}_{1}+\tilde{V}_{2}}{2}
$$

where, $\tilde{U}_{1}=\tilde{U}_{2}=U$ and $\tilde{V}_{1}=\tilde{V}_{2}=V$ for the $\vee$-probe because both wires 1 and 2 are in the same homogeneous $x$ - $y$-plane, whereas for the $\times$-probe $\tilde{U}_{1}=U_{z_{1}}, \tilde{U}_{2}=U_{z_{2}}$ because wires 1 and 2 are in different homogeneous planes, and similarly for $V$-velocity. Wires 1 and 2 at different $z$-locations correspond to the mean velocities $U_{1}$ and $U_{2}$ (see the inset in figure $14(\mathrm{c}))$. For the $\times$-probe, $U$ is under-predicted because according to (21) the 'measured' velocity is the mean of $U_{1}$ and $U_{2}$ which is less than the actual $U$, as illustrated in the inset of figure 15(c). Similarly errors in $V$ occur because of the differences in $U_{1}$ and $U_{2}$. (Note that in the present case wire 1 is considered to be below wire 2 and that is the reason for negative $\tilde{V}$, or else it would have the same magnitude but a positive error.) Also noticeable are the errors in the mean spanwise velocities close to the wall measured by the $\times$-probe in figure $14(\mathrm{~d})$ compared to the non-existence of errors in the $\vee$-probes.

It will be shown in the next section that the qualitative trends for the errors in the measurements observed in the DNS simulations of the $\vee$ and $\times$-probes of a low $R e_{\tau}$ are similar to those observed in actual experiments at a higher $R e_{\tau}$. 

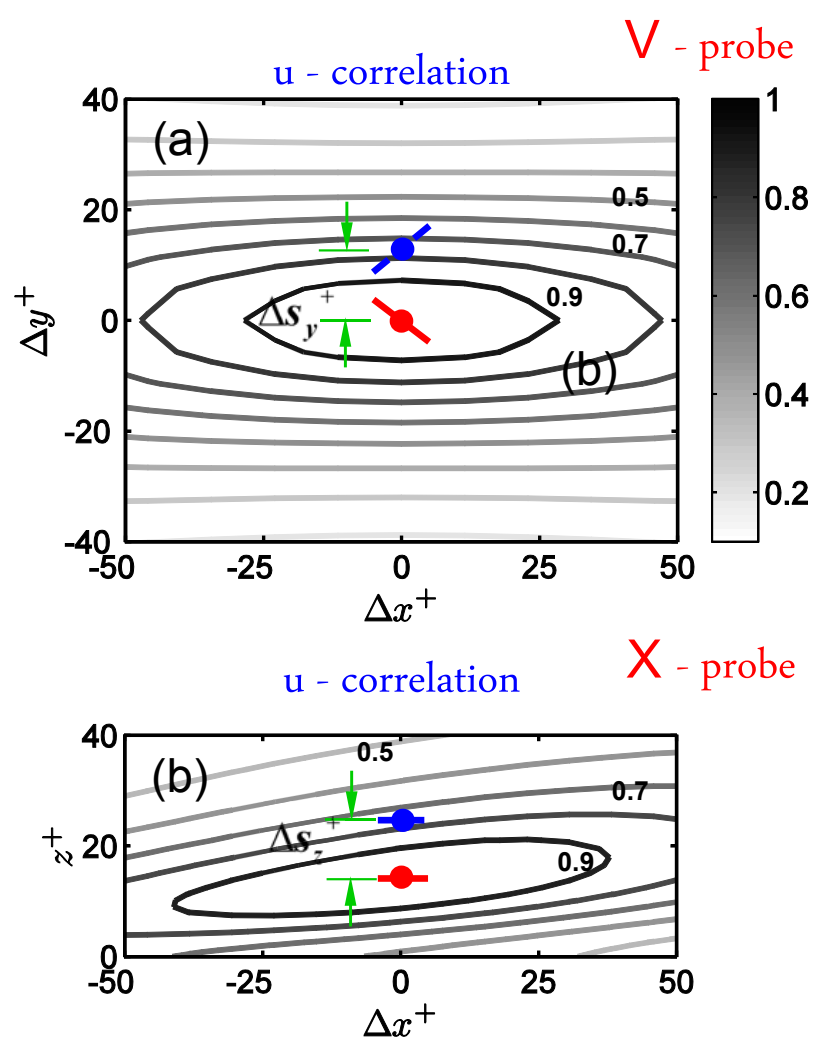

Figure 15. $R_{u u}$ for $\vee$ and $\times$-probe at the nominal wire location of $z^{+}=20$. Also shown are the schematics of wires with a spacing of $\approx 12$. (a) $R_{u u}$ for $\vee$-probe. (b) $R_{u u}$ for $\times$-probe.

\section{Some experimental results}

Here we present an example of both the $\vee$ - and $\times$-probe measurements taken at $R e_{\tau} \approx 5000$ in the High Reynolds Number Turbulent Boundary Layer Wind Tunnel (HRNBLWT) located at the University of Melbourne. This facility has a $27 \mathrm{~m}$ working section which allows a thick boundary layer to be developed, permitting relatively high Reynolds numbers while keeping the free-stream velocity relatively low. This 'big and slow' approach allows velocity measurements with relatively good spatial resolutions. The probes prongs are built with stainless steel and Platinum is used as the sensing element (with a wire diameter of $2.5 \mu \mathrm{m})$. The same wire length $\left(l^{+} \approx 15\right)$ is used for all the probes. Wire spacings are $\Delta s_{y}^{+} \approx 15$ for the $\vee$-probe and $\Delta s_{z}^{+} \approx 5$ for the $\times$ probe. These dimensions in inner-viscous unit are not far from those used in the DNS simulations discussed in the previous sections, and are reasonably simple to manufacture in the laboratory using conventional techniques.

Figures 16(a) and (b) show the streamwise $\left(\bar{U}^{+}\right)$and spanwise $\left(\bar{V}^{+}\right)$mean velocity profiles, respectively, from the $\vee$ - (red circles) and $\times$-probes (blue squares). For the $\times-$ probe, $\bar{U}^{+}$is slightly less than that measured by the $\vee$-probe for smaller $z^{+}$. Likewise, $\bar{V}^{+}$for the $\vee$-probe is quite close to zero while the same result from the $\times$-probe is strongly negative close to the wall. These trends are as predicted from the analysis of 

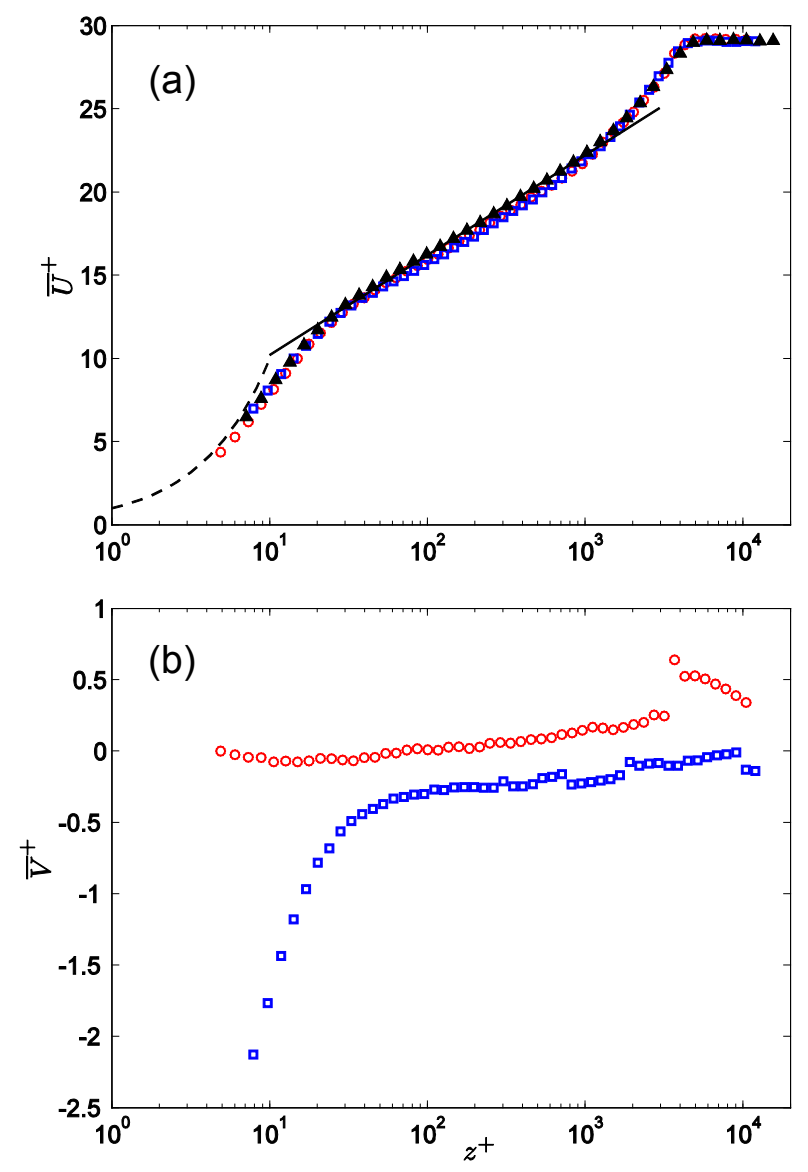

Figure 16. Mean velocities at $R e_{\tau}=5000$, experimentally measured with a $\vee$-probe (red circles) and an $\times$-probe (blue squares). (a) Streamwise velocities. (b) Spanwise velocities. Filled (triangle) symbols in (a) are from a single-wire measurement at comparable $R e_{\tau}$ [Kulandaivelu, 2012]. The dashed line in (a) is $z^{+}=U^{+}$, and the solid line is $U^{+}=1 / 0.39 \log \left(z^{+}\right)+4.3$ [Marusic et al., 2013].

the DNS and from the modelled probes.

The variances, $\bar{u}_{r m s}^{2+}$ and $\bar{v}_{r m s}^{2+}$, are shown in figures $17(\mathrm{a})$ and (b) for $\vee$ (red circles) and $\times$-probes (blue squares), respectively. The spanwise variance, $\bar{v}_{r m s}^{2+}$, is over-predicted for the $\vee$-probe compared to the $\times$-probe, whereas for $\bar{u}_{r m s}^{2+}$ both probes seem to produce similar results. These trends are similar to those found from the DNS in the previous sections. The increased $v_{r m s}^{2+}$ errors close to the wall for the $\vee$-probe compared to the $\times$-probe are also noticed by Österlund [1999] from his measurements in a turbulent boundary layer. Note that in the present case $\Delta s_{z}^{+}$for the $\times$-probe is smaller than $\Delta s_{y}^{+}$ for the $\vee$-probe and this has reduced the error in $\bar{u}_{r m s}^{2+}$, which should have been present for the $\times$-probe if $\Delta s_{z}^{+}$was equal to $\Delta s_{y}^{+}$. This also highlights the fact that it is more practical to make $\times$-probes with smaller wire spacing $\left(\Delta s_{z}^{+}\right)$than to do so for $\vee$-probes (where the minimum $\Delta s_{y}^{+} \approx l^{+}$). Note that here we can only discuss the relative errors between the $\times$ - and $\vee$-probes since we have no 'correct' results to compare to. However it is not difficult to gauge from extrapolating the low $R e$ trends (as well as the works 

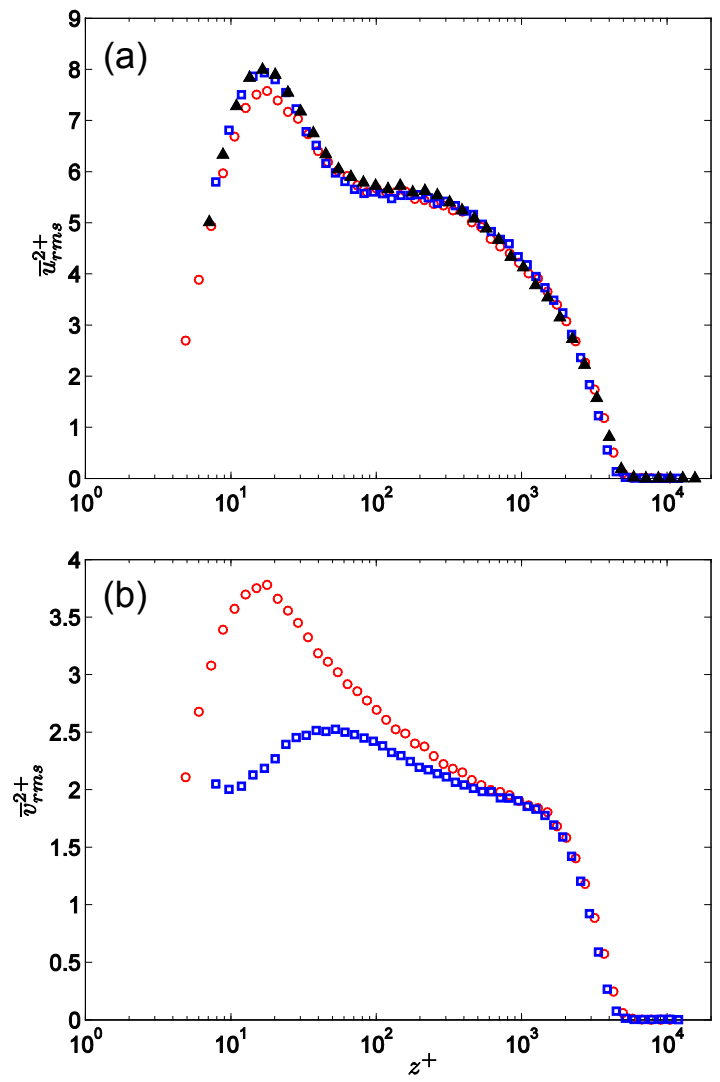

Figure 17. Variances at $R e_{\tau}=5000$, experimentally measured with a $\vee$-probe (red circles) and an $\times$-probe (blue squares). (a) Streamwise variance, $\bar{u}_{r m s}^{2+}$. (b) Spanwise variance, $\bar{v}_{r m s}^{2+}$. Filled (triangle) symbols in (a) are from a single-wire measurement at comparable $R e_{\tau}$ [Kulandaivelu, 2012].

of other researchers, e.g., Österlund, 1999) that the $\times$-probe performs better than the $\vee$-probe, at least in this case.

\section{Summary and Conclusions}

The effects induced by the finite dimension of $\vee$-probes and $\times$-probes on the measured means and variances of the streamwise-spanwise velocities in wall-bounded turbulent flows are investigated using the DNS database of del Álamo et al. [2003] at $R e_{\tau}=934$, as well as employing analytical tools. Experimental results are also presented corroborating the conclusions drawn and showing the practicality of the study.

It is not uncommon to view $\vee$-probes as 'simpler' because the mean flow does not vary over the entire sensor, whereas both the wires of an $\times$-probe (configured to measure the streamwise spanwise components) experience different velocities due to the velocity gradient in turbulent flows. However, it shown that for linear filters (which are good approximations to the hot-wire filtering), the means and the variances 'decouple', i.e., the measured mean for both $\vee$ and $\times$-probes are affected only by the actual mean velocities, whereas variances are not affected by the mean velocities, but only by the 

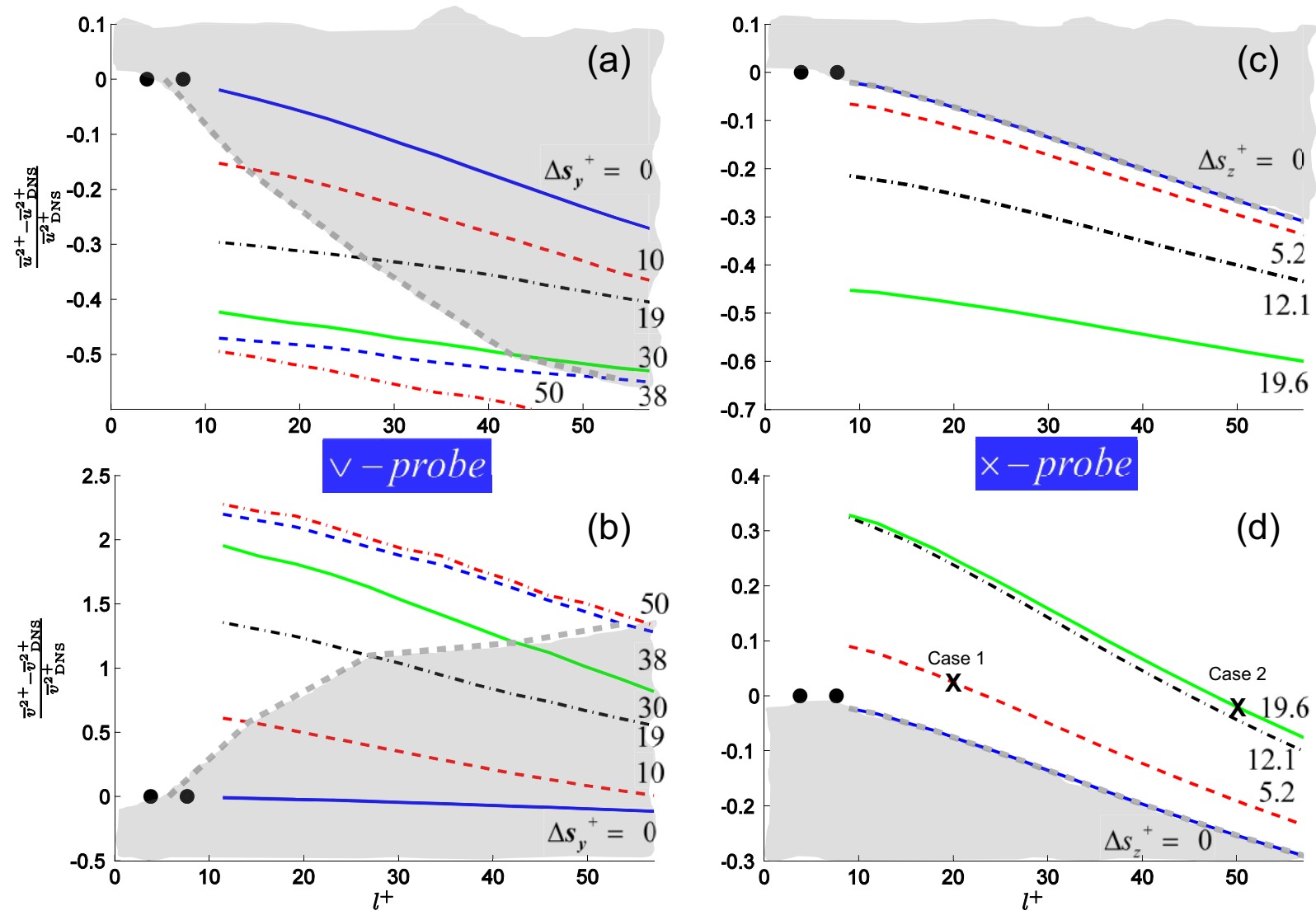

Figure 18. Distributions of the relative difference $(\mathcal{R D})$ for various configurations of $\vee$ and $\times$-probes at $z^{+}=15$ and $\theta=45^{\circ}$ from the un-averaged DNS data. (a) $\mathcal{R} \mathcal{D}_{u}$ for $\vee$-probes. (b) $\mathcal{R} \mathcal{D}_{v}$ for $\vee$-probes. (c) $\mathcal{R} \mathcal{D}_{u}$ for $\times$-probes. (d) $\mathcal{R} \mathcal{D}_{v}$ for $\times$-probes. Also shown here are two crosses marked case 1 and 2 which are studied in detail in figure 19. The grey area separated by thick grey lines show regions which correspond to probes which are physically infeasible to manufacture.

two-point velocity correlations over the wire spacing $\Delta s_{y}$ or $\Delta s_{z}$. Thus, both $\vee$ and $\times-$ probes can be analysed under the same framework. This immediately highlights that for the $\vee$-probe the mean velocity measured is the same as the actual velocity, whereas for the $x$-probe this is not the case, although this under-prediction is small and generally limited to the near-wall region. More noticeably, the $\times$-probe also induces errors in the measured mean spanwise velocity $\bar{V}^{+}$(which should be exactly zero in channel flows and close to zero in TBLs). Generally $\times$-probes under-predict the mean streamwise velocities whereas the spanwise velocities are either under or over-predicted depending on the orientation of the wires.

Here we note that the wires for both $\vee$ - and $\times$-probes for stream-spanwise measurements are each in a plane with the same mean velocity, which is referred to here as the homogenous plane. This is in contrast to uw (stream-wallnormal) velocity measurements where the wires experience a mean shear, i.e. they reside in the inhomogeneous plane/s of the flow.

Unlike the mean quantities, the variances are more complicated functions of the 
probe parameters. There are three parameters which can be used to categorize $\vee$ and $\times$-probes, namely, the inclination angle of the wires with respect to the streamwise velocity, $\theta\left(0^{\circ}\right.$ when the wire is aligned with the streamwise direction and $90^{\circ}$ for spanwise alignment), the length of the wires (assuming both the wires are of the same length), $l$, and the spacing between the wires, $\Delta s_{y}$ (for $\vee$-probes), or $\Delta s_{z}$ (for $\times$-probes). The effect of $l$ is well understood from the study of single wires in Philip et al. [2013], which shows that increasing $l$ always decreases the variances. To isolate the effect of $\theta$ from $\Delta s$, a single inclined wire is studied (in the homogeneous plane), where the only parameters are $\theta$ and $l$. It is found that the averaged $u_{r m s}, \tilde{u}_{r m s}$ is highly attenuated between $\theta=90^{\circ}$ to $\approx 45^{\circ}$, and thereafter a quick decrease in attenuation when the wire is along the streamwise direction (although realistically a wire aligned with the flow would be useless due to the aerodynamic interference from the prongs). This has been shown as the effect of the two-point correlations (figure 3(b)). A simple model of the correlation (in equation (4)) is able to estimate these effects found in the DNS simulations (figure 2).

The effects of $l, \theta$ and $\Delta s$ on $u_{r m s}$ and $v_{r m s}$ are studied in detail in figures 5 and 11 for the $\vee$ - and $\times$-probes, respectively at $z^{+} \approx 15$. Since the effect of $\theta$ is generally the same as that found in the analysis of the single inclined wire, a single angle $\theta=45^{\circ}$ is isolated and studied in detail. This is also the most commonly used configuration in laboratories. Figures 18(a, b) and (c,d) show the data from figures 6 and 12, respectively, for $\vee$ - and $\times$-probes at $\theta=45^{\circ}$ and at $z^{+} \approx 15$ as an error, or as a relative difference $(\mathcal{R D})$ from the actual DNS data i.e., $\left(\bar{u}^{2+}-\bar{u}_{\text {DNS }}^{2+}\right) / \bar{u}_{\text {DNS }}^{2+}=: \mathcal{R} \mathcal{D}_{u}$ for streamwise velocity, and a similar definition for spanwise velocity. Note that some extra simulations at different $\Delta s_{y}^{+}$are also included in figures $18(\mathrm{a}, \mathrm{b})$ for clarification. For $\vee$ and $\times$-probes, for both $u_{r m s}$ and $v_{r m s}$, an increase in $l^{+}$is always accompanied by a decrease in $\mathcal{R} \mathcal{D}$, which is the effect of spatial averaging along the wire. For the same $\Delta s$ the absolute value of $\mathcal{R} \mathcal{D}_{u}$ for the $\vee$-probe is less than that of the $\times$-probe, owing to the faster drop of $u$-correlation along the wall-normal direction compared to the spanwise direction (c.f. figure 13(a,c)). The values of $\mathcal{R} \mathcal{D}_{v}$ are mostly positive for the $\vee$-probe because the measured velocity $\bar{v}$ is the difference of two large numbers $u$ from the two wires (after neglecting $v$ from equation (14)), and consequently the measured variance is much higher than the actual variance. Since with increasing $l^{+}, \mathcal{R} \mathcal{D}_{v}$ decreases; this combined effect of $l^{+}$and $\Delta s_{y}^{+}$ results in the distribution shown in figure $18(\mathrm{~b})$.

In the case of the $\times$-probe for $\mathcal{R} \mathcal{D}_{v}$, yet another effect comes into play - the averaging effect of $v$ (since, now unlike in the $\vee$-probe we cannot neglect the effect of $v$ from equation (14), because the $v$ correlations are much smaller than unity), which tends to decrease $\mathcal{R} \mathcal{D}_{v}$. Thus, for the $\times$-probe there are two effects which decrease $\mathcal{R} \mathcal{D}_{v}$ and one that increases it (see equation (19)), and this makes the reduction of $\mathcal{R} \mathcal{D}_{v}$ (or reduction in errors) an efficient process in $\times$-probes compared to $\vee$-probes.

These competing effects can even make the error vanish in $\vee$-probes with increasing $l^{+}$and more so in $\times$-probes (see, figures $13(\mathrm{~b}, \mathrm{~d})$ ), which of course does not mean that there are no finite dimension errors. To illustrate this point, consider two cases 
corresponding to the $\times$-probes shown in figure $13(\mathrm{~d})$ by two crosses and marked case 1 and 2. Case 1 has $l^{+}=20$ and $\Delta s_{z}^{+}=5.2$, and case 2 has $l^{+}=50$ and $\Delta s_{z}^{+}=19.6$. According to figure $13(\mathrm{~d})$ both have approximately the same $\mathcal{R} \mathcal{D}_{v}$, which might suggest that both configurations are equally good for measuring $v$. However, this is not completely correct and can be observed in figure 19, which shows the fluctuating $v$ signal for the actual DNS, as well as cases 1 and 2. It is evident that the smaller configuration (case 1) follows the DNS much more closely than case 2 even though all three have similar variances. A better measure of the error could be defined as the normalised variance of the difference between the DNS $\left(v_{0}^{\prime+}\right)$ and the measured $\left(v_{1}^{\prime+}\right.$ for case 1) signal:

$$
\mathcal{E}_{1}:=\frac{\left\langle\left(v_{0}^{\prime+}-v_{1}^{\prime+}\right)^{2}\right\rangle}{\left\langle\left(v_{0}^{\prime+}\right)^{2}\right\rangle}=\mathcal{R} \mathcal{D}_{v}+2\left(1-\frac{R_{v_{0} v_{1}}}{\left\langle\left(v_{0}^{\prime+}\right)^{2}\right\rangle}\right)
$$

where, $R_{v_{0} v_{1}}:=\left\langle\left(v_{0}^{\prime+} v_{1}^{\prime+}\right)^{2}\right\rangle$ is the correlation coefficient between the DNS and the measured signal, and in the above notations, $\mathcal{R} \mathcal{D}_{v}=\left\langle v_{1}^{+2}\right\rangle /\left\langle v_{0}^{\prime+2}\right\rangle-1$. A similar definition can be written for case 2. Note that if the DNS and the measured signals are perfectly correlated then the error reduces to the earlier mentioned $\mathcal{R} \mathcal{D}_{v}$, which is not always the case. In the present example, even though $\mathcal{R} \mathcal{D}_{v}$ for both case 1 and 2 is about the same (0.0100 and -0.0358 , respectively), $\mathcal{E}_{1}=0.1937$ (for case 1 ), whereas, $\mathcal{E}_{2}=1.0236$ (for case 2 ). This shows that case 1 has a better correlation with the DNS (as is evident when observing figure 19) than case 2 and consequently case 1 has a much lower $\mathcal{E}$ compared to case 2 . These two examples point to the fact that even though $\mathcal{R} \mathcal{D}$ is the most common measure of error used in the literature for studying spatial averaging of sensors, and even though a good representative, one has to be cautious. A complete measure of errors might even require considering higher order statistics depending upon the specific need.

From a practical point of view for a $\vee$-probe not all $\Delta s_{y}$ are feasible. For instance, in the case of $\theta=45^{\circ}, \Delta s_{y}$ cannot be smaller than $l / \sqrt{2}$, without the two wires occupying the same physical space, which is impossible. This constraint is shown in figures 18(a,b) with a thick (grey) dashed line. The analogous constraint for $\times$-probes is the positivity of $\Delta s_{z}$, which is shown similarly in figures $18(\mathrm{c}, \mathrm{d})$. The grey shaded areas in the figures are the 'inaccessible regions' due to physical constants. Practically it suggests that for a given wire length the best option (i.e. least errors) is attained by remaining as close as possible to the grey dashed lines. It is clear that there is much more flexibility with an $\times$-probe to reduce errors. This is evident from the experimental results shown in the previous section where it was easier to make an $\times$-probe with a smaller $\Delta s$ than a $\vee$-probe. Increasing $z$ makes the $\times$-probe more accurate for $u_{r m s}$ (c.f. figure 15 and also $17(\mathrm{a}))$ due to the increased $u$-correlation in $z$-direction for higher $z$. In all, it appears that $\times$-probes are better suited for $u-v$ (stream-spanwise velocity) measurements than $\vee$-probes. 


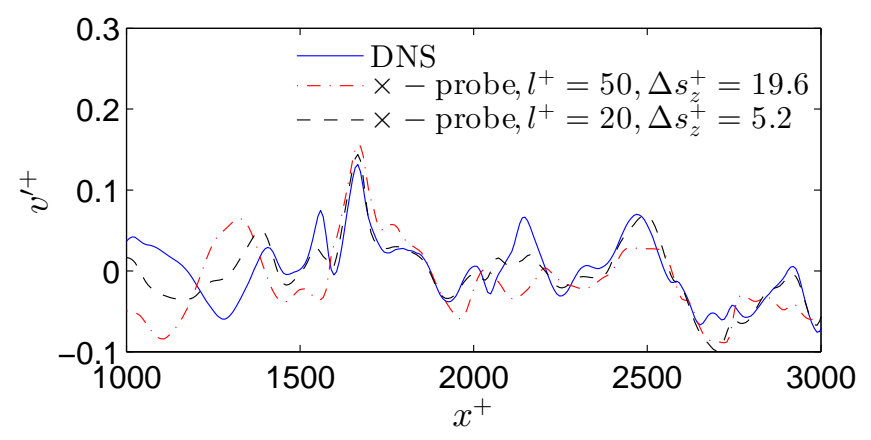

Figure 19. Spanwise fluctuating velocity at $z^{+}=15$ from $\times$-probes. The solid line is the actual DNS signal; the dashed line corresponds to case $1\left(l^{+}=20\right.$ and $\left.\Delta s_{z}^{+}=5.2\right)$, and the dashed-dotted line corresponds to case $2\left(l^{+}=50\right.$ and $\left.\Delta s_{z}^{+}=19.6\right)$. Cases 1 and 2 are indicated in figure 18(d) by crosses.

\section{Acknowledgments}

The authors gratefully acknowledge support from the Australian Research Council.

\section{Appendix: Derivation of the expressions for filtered variances}

Starting with equation (14) for the 'measured' or filtered fluctuating velocities, $\overline{u^{\prime}}$ and $\overline{v^{\prime}}$, the variances can be found by squaring them and taking an ensemble average, which results in,

$$
\begin{aligned}
& \left\langle\overline{u^{\prime}} \overline{u^{\prime}}\right\rangle=\frac{1}{4}\left(\left\langle\tilde{u}_{1}^{\prime} \tilde{u}_{1}^{\prime}\right\rangle+\left\langle\tilde{u}_{2}^{\prime} \tilde{u}_{2}^{\prime}\right\rangle+2\left\langle\tilde{u}_{1}^{\prime} \tilde{u}_{2}^{\prime}\right\rangle+\right. \\
& \left.\left\langle\tilde{v}_{1}^{\prime} \tilde{v}_{1}^{\prime}\right\rangle+\left\langle\tilde{v}_{2}^{\prime} \tilde{v}_{2}^{\prime}\right\rangle-2\left\langle\tilde{v}_{1}^{\prime} \tilde{v}_{2}^{\prime}\right\rangle\right),
\end{aligned}
$$

and,

$$
\begin{aligned}
& \left\langle\overline{v^{\prime}}{\overline{v^{\prime}}}\right\rangle=\frac{1}{4}\left(\left\langle\tilde{u}_{1}^{\prime} \tilde{u}_{1}^{\prime}\right\rangle+\left\langle\tilde{u}_{2}^{\prime} \tilde{u}_{2}^{\prime}\right\rangle-2\left\langle\tilde{u}_{1}^{\prime} \tilde{u}_{2}^{\prime}\right\rangle+\right. \\
& \left.\left\langle\tilde{v}_{1}^{\prime} \tilde{v}_{1}\right\rangle+\left\langle\tilde{v}_{2}^{\prime} \tilde{v}_{2}^{\prime}\right\rangle+2\left\langle\tilde{v}_{1}^{\prime}{ }_{1} \tilde{v}_{2}^{\prime}\right\rangle\right),
\end{aligned}
$$

where, $\tilde{u}_{1}^{\prime}$ and $\tilde{v}_{1}^{\prime}$ are the fluctuating velocities averaged over wire 1 over its length, and similarly $\tilde{u}_{2}^{\prime}$ and $\tilde{v}_{2}^{\prime}$ for wire 2 . Furthermore, the instantaneous velocity fluctuations are (as mentioned in the main text), $u_{1}^{\prime}$ and $v_{1}^{\prime}$. Notations can be simplified by denoting the measured variance by, $\bar{u}_{r m s}^{2}:=\left\langle\overline{u^{\prime}} \overline{u^{\prime}}\right\rangle$ and $\bar{v}_{r m s}^{2}:=\left\langle\overline{v^{\prime}} \overline{v^{\prime}}\right\rangle$.

For $\vee$-probes both wires 1 and 2 are located on the same horizontal plane and if we consider both wires to be of the same length, $\left(l_{1}^{+}=l_{2}^{+}=l^{+}\right),\left\langle\tilde{u}_{1}^{\prime} \tilde{u}_{1}^{\prime}\right\rangle=\left\langle\tilde{u}_{2}^{\prime} \tilde{u}_{2}^{\prime}\right\rangle=: \tilde{u}_{r m s}^{2}$, and in a similar way, $\left\langle{\tilde{v^{\prime}}}_{1}{\tilde{v^{\prime}}}_{1}\right\rangle=\left\langle{\tilde{v^{\prime}}}_{2}{\tilde{v^{\prime}}}_{2}\right\rangle=$ : $\tilde{v}_{r m s}^{2}$. The correlation, $\left\langle\tilde{u}_{1}^{\prime} \tilde{u}_{2}^{\prime}\right\rangle / \tilde{u}_{r m s}^{2}$, depends on the spacing between the two wires, $\Delta s_{y}$ along the $y$-direction, and is denoted by, $\tilde{R}_{N_{u u}}\left(\Delta s_{y}\right)$. Since the correlation between the velocities that are already averaged over the wire length, $l$, strictly, $\tilde{R}_{N_{u u}}\left(\Delta s_{y} ; l\right)$ as mentioned in the main text. Similarly, $\left\langle\tilde{v}^{\prime}{ }_{1} \tilde{v}_{2}^{\prime}\right\rangle / \tilde{v}_{r m s}^{2}=: \tilde{R}_{N_{v v}}\left(\Delta s_{y}\right)$. Furthermore, the wire-averaged variances (e.g. $\tilde{u}_{r m s}^{2}$ ) can be related to the actual variances $\left(u_{r m s}^{2}\right)$ using equation (3). Approximating the $u$ 
correlations with the model (4), leads to equation (7), or, $\tilde{u}_{r m s}^{2} / u_{r m s}^{2}=\mathrm{F}_{u}$, where, $\mathrm{F}_{u}$ is the expression on the right-hand-side of (7). Similarly, with an appropriate model for $v$-correlations, in symbolic form we can write, $\tilde{v}_{r m s}^{2} / v_{r m s}^{2}=\mathrm{F}_{v}$. Substituting these definitions into the above two equations leads to,

$$
\begin{aligned}
& \frac{\bar{u}_{r m s}^{2}}{u_{r m s}^{2}} \approx \frac{\mathrm{F}_{u}}{2}\left(1+\tilde{R}_{N_{u u}}\left(\Delta s_{y}\right)\right)+ \\
& \frac{\mathrm{F}_{v}}{2}\left(1-\tilde{R}_{N_{v v}}\left(\Delta s_{y}\right)\right) \frac{v_{r m s}^{2}}{u_{r m s}^{2}},
\end{aligned}
$$

and,

$$
\begin{aligned}
& \frac{\bar{v}_{r m s}^{2}}{v_{r m s}^{2}} \approx \frac{\mathrm{F}_{v}}{2}\left(1+\tilde{R}_{N_{v v}}\left(\Delta s_{y}\right)\right)+ \\
& \frac{\mathrm{F}_{u}}{2}\left(1-\tilde{R}_{N_{u u}}\left(\Delta s_{y}\right)\right) \frac{u_{r m s}^{2}}{v_{r m s}^{2}} .
\end{aligned}
$$

Three points are noted: (i) if the spacing between the wires is zero (i.e., $\Delta s_{y}=0$ ), $\tilde{R}_{N}=1$ and the above expressions reduce to that for simple averaging. On the other hand if the wire lengths are assumed to be zero, $\mathrm{F}_{u}=\mathrm{F}_{v}=1$, and we recover the expressions by [Suzuki and Kasagi, 1992] originally derived for an $X$-probe measuring $u-w$ velocities. (ii) with the help of the above expressions, once the measured variance $\bar{u}_{r m s}^{2}$ or $\bar{v}_{r m s}^{2}$ is known the corresponding actual variances, $u_{r m s}^{2}$ or $v_{r m s}^{2}$ can be estimated, or vice-versa. This is the way in which the symbols in figure 6 are generated. (iii) we have assumed in the above (and throughout this work) that the two wires are of the same length, which also makes the inversion of the above expressions possible.

Some simplifications can be made in (25) by noting that $v_{r m s}^{2} / u_{r m s}^{2}$ is small compared to the other terms. Therefore, the second term on the right hand side of (25) can be neglected which results in:

$$
\frac{\bar{u}_{r m s}^{2}}{u_{r m s}^{2}} \approx \frac{\mathrm{F}_{u}}{2}\left(1+\tilde{R}_{N_{u u}}\left(\Delta s_{y}\right)\right),
$$

which is the same as eqn (15). Furthermore, $\tilde{R}_{N_{v v}}\left(\Delta s_{y}\right)$ hardly drops with $\left(\Delta s_{y}\right)$ and its value can be approximated by 1 (see figures $13(\mathrm{~b})$ and (d), which are essentially for $R_{N_{v v}}$, however, $\tilde{R}_{N_{v v}}$ is not very different, as mentioned in reference to figure 10$)$. Therefore, assuming $\tilde{R}_{N_{v v}}\left(\Delta s_{y}\right)=1$ in (26),

$$
\frac{\bar{v}_{r m s}^{2}}{v_{r m s}^{2}} \approx \mathrm{F}_{v}+\frac{\mathrm{F}_{u}}{2}\left(1-\tilde{R}_{N_{u u}}\left(\Delta s_{y}\right)\right) \frac{u_{r m s}^{2}}{v_{r m s}^{2}} .
$$

Substituting $u_{r m s}^{2}=\tilde{u}_{r m s}^{2} / \mathrm{F}_{u}$ and $v_{r m s}^{2}=\tilde{v}_{r m s}^{2} / \mathrm{F}_{v}$ in the above equation leads to (16).

For the $\times$-probe, we again start from equations (23) and (24). In this case wires 1 and 2 are located on two different horizontal planes and consequently, $\left\langle{\tilde{u^{\prime}}}_{1}{\tilde{u^{\prime}}}_{1}\right\rangle \neq$ $\left\langle\tilde{u}_{2}^{\prime} \tilde{u}_{2}^{\prime}\right\rangle$, and, $\left\langle\tilde{v}_{1}^{\prime} \tilde{v}_{1}^{\prime}\right\rangle \neq\left\langle\tilde{v}_{2}^{\prime} \tilde{v}_{2}^{\prime}{ }_{2}\right\rangle$. Furthermore, the velocity that needs to be inferred is at the middle of the two wires. Without loss of generality, consider wire 1 to be located below and wire 2 to be located at a distance $\Delta s_{z}$ above wire 1, such that the inferred velocity, say $u_{0}, v_{0}$, is at a distance $\Delta s_{z} / 2$ (say, $z=z_{0}$ ) above wire 1 (c.f. figure $1(\mathrm{c})$ ). 
Since the variances are smooth functions of the wall-normal distance, $z$, using Taylor expansion, we can write,

$$
\begin{aligned}
& \left\langle\tilde{u}_{1}^{\prime} \tilde{u}_{1}^{\prime}\right\rangle=\left\langle\tilde{u}_{0}^{\prime} \tilde{u}_{0}^{\prime}\right\rangle-\left.\left(\frac{\Delta s_{z}}{2}\right) \frac{\mathrm{d}\left\langle\tilde{u^{\prime}} \tilde{u}^{\prime}\right\rangle}{\mathrm{d} z}\right|_{z_{0}}+ \\
& \left.\left(\frac{\Delta s_{z}}{2}\right)^{2} \frac{1}{2 !} \frac{\mathrm{d}^{2}\left\langle\tilde{u^{\prime}} \tilde{u}^{\prime}\right\rangle}{\mathrm{d} z^{2}}\right|_{z_{0}}-\cdots,
\end{aligned}
$$

and similarly for $\left\langle\tilde{u}_{2}^{\prime} \tilde{u}_{2}{ }_{2}\right\rangle$, where $\left\langle\tilde{u}_{0}^{\prime} \tilde{u}_{0}^{\prime}\right\rangle$ is $\left\langle\tilde{u^{\prime}} \tilde{u^{\prime}}\right\rangle$ at $z=z_{0}$. Substituting them in equations (23) and (24) and neglecting the terms of order $\left(\Delta s_{z}\right)^{2}$ and higher, we obtain,

$$
\begin{aligned}
& \frac{\bar{u}_{r m s}^{2}}{u_{r m s}^{2}} \approx \frac{\mathrm{F}_{u_{0}}}{2}\left(1+\tilde{R}_{N_{u u}}\left(\Delta s_{z}\right)\right)+ \\
& \frac{\mathrm{F}_{v_{0}}}{2}\left(1-\tilde{R}_{N_{v v}}\left(\Delta s_{z}\right)\right) \frac{v_{r m s}^{2}}{u_{r m s}^{2}}
\end{aligned}
$$

and,

$$
\begin{aligned}
& \frac{\bar{v}_{r m s}^{2}}{v_{r m s}^{2}} \approx \frac{\mathrm{F}_{v_{0}}}{2}\left(1+\tilde{R}_{N_{v v}}\left(\Delta s_{z}\right)\right)+ \\
& \frac{\mathrm{F}_{u_{0}}}{2}\left(1-\tilde{R}_{N_{u u}}\left(\Delta s_{z}\right)\right) \frac{u_{r m s}^{2}}{v_{r m s}^{2}},
\end{aligned}
$$

where, $u_{r m s}^{2}:=\left\langle u_{0}^{\prime} u_{0}^{\prime}\right\rangle, F_{u_{0}}:=\left\langle\tilde{u}_{0}^{\prime} \tilde{u}_{0}^{\prime}\right\rangle /\left\langle u_{0}^{\prime} u_{0}^{\prime}\right\rangle, \tilde{R}_{N_{u u}}\left(\Delta s_{z}\right)$ is the normalised $u$ correlation between $\tilde{u}_{1}$ and $\tilde{u}_{2}$ located at wire 1 and 2 , respectively, separated by $\Delta s_{z}$, and similar definitions hold for the $v$ component terms. The above equations show that for $\times$-probes we obtain expressions similar to that of $\vee$-probes, if terms of order $\left(\Delta s_{z}\right)^{2}$ and higher are neglected. It is estimated that at about $z^{+}=15$ (where the errors in $u_{r m s}^{2}$ are the largest), $\left(\Delta s_{z}\right)^{2}$ terms are about $1 \%$ of the $\left(\Delta s_{z}\right)$ terms if $\left(\Delta s_{z}^{+}\right) \lesssim 15$, which covers much of the practical range for $\times$-probes.

Simplifications for (30) can be obtained by neglecting the term involving $v_{r m s}^{2} / u_{r m s}^{2}$ and retaining the second term in (31). This gives a similar relation for $\bar{u}_{r m s}^{2}$ as in $V$ probes and leads to equation (18). However, an approximation similar to that used for $\vee$-probes in $\bar{v}_{r m s}^{2}$ is not possible for $\times$-probes because $\tilde{R}_{N_{v v}}$ drops much faster in $z$ than in $y$ (c.f., figures $13(\mathrm{~b})$ and $(\mathrm{d})$ ), and $\tilde{R}_{N_{v v}}$ cannot be substituted for 1 since it is much smaller than 1 . This enforces us to keep all the terms in (31) and now $\tilde{R}_{N_{v v}}$ needs to be modelled or known. This is the main cause for the differences observed between the errors in $\times$ and $\vee$-probes. Equation (31) becomes (19) after substituting $u_{r m s}^{2}=\tilde{u}_{r m s}^{2} / \mathrm{F}_{u}$ and $v_{r m s}^{2}=\tilde{v}_{r m s}^{2} / \mathrm{F}_{v}$.

\section{References}

M. Abramowitz and I. A. Stegun. Handbook of Mathematical Functions with Formulas, Graphs, and Mathematical Tables. Dover, New York, ninth dover printing, tenth gpo printing edition, 1964.

H. H. Bruun. Hot-wire anemometry. Principles and Signal Analysis. Oxford University Press, 1995. 
C. C. Chin, N. Hutchins, A. S. H. Ooi, and I. Marusic. Use of direct numerical simulation (DNS) data to investigate spatial resolution issues in measurements of wall-bounded turbulence. Meas. Sci. Technol., 20:115401, 2009.

J. M Österlund. Experimental Studies of Zero Pressure-Gradient Turbulent BoundaryLayer Flow. PhD thesis, KTH, Stockholm, 1999.

J. C. del Álamo, J. Jiménez, P. Zandonade, and R. D. Moser. Scaling of the energy spectra of turbulent channels. J. Fluid. Mech, 490:37-74, 2003.

H. J. Hussein, S. P. Capps, and W. K. George. Velocity measurements in a highReynolds-number, momentum-conserving, axisymmetric, turbulent jet. J. Fluid. Mech, 258:31-75, 1996.

N. Hutchins, T. B. Nickels, I. Marusic, and M. S. Chong. Hot-wire spatial resolution issues in wall-bounded turbulence. J. Fluid. Mech, 635:103-136, 2009.

P. Krogstad and P. E. Skåte. Influence of a strong adverse pressure gradient on the turbulent structure in a boundary layer. Phys. Fluids, 7:2014-2024, 1995.

V. Kulandaivelu. Evolution of zero pressure gradient turbulent boundary layers from different initial conditions. PhD thesis, The University of Melbourne, Melbourne, 2012.

P. M. Ligrani and P. Bradshaw. Spatial resolution and measurement of turbulence in the viscous sublayer using subminiature hot-wire probes. Exp. Fluids, 5:407-417, 1987.

I. Marusic and A. E. Perry. A wall-wake model for the turbulence structure of boundary layers. Part 2. Further experimental support. J. Fluid. Mech, 298:389-407, 1995.

I. Marusic, J. P. Monty, M. Hultmark, and A. J. Smits. On the logarithmic region in wall turbulence. J. Fluid. Mech, 716, R3:doi:10.1017/jfm.2012.511, 2013.

P. Moin and P. R. Spalart. Contributions of numerical simulation data bases to the physics, modeling, and measurement of turbulence. Technical report, NASA Tech. Memo 100022, 1987.

T. B. Nickels and A. E. Perry. An experimental and theoretical study of the turbulent coflowing jet. J. Fluid. Mech, 309:157-182, 1996.

A. E. Perry. Hot-wire anemometry. Oxford University Press, 1982.

A. E. Perry, K. L. Lim, and S. M. Henbest. An experimental study of the turbulence structure in smooth- and rough-wall boundary layers. J. Fluid. Mech, 177:437-466, 1987.

J. Philip, N. Hutchins, J. P. Monty, and I. Marusic. Spatial averaging of velocity measurements in wall-bounded turbulence: General considerations and single hotwires. submitted to Meas. Sci. Technol., 2013.

Y. Suzuki and N. Kasagi. Evaluation of hot-wire measurements in wall shear turbulence using a direct numerical simulation database. Exp. Therm. Fluid Sci., 5:69-77, 1992.

M. Vallikivi, M. Hultmark, and A. J. Smits. Turbulence measurements at high reynolds numbers using a new inclined nano-scale thermal anemometry probe. In P. A. 
Brandner and B. W. Pearce, editors, Proceedings of the Eighteenth Australasian Fluid Mechanics Conference, volume 18, pages 179-182, Launceston, Australia, 37 December 2012.

P. V. Vukoslavčević and J. M. Wallace. On the accuracy of simultaneously measuring velocity component statistics in turbulent wall flows with arrays of three or four hotwire sensors. Exp. Fluids, 51:1509-1519, 2011. 